\title{
Effects of Garlic Allium sativum Powder on Nutrient Digestibility, Haematology, and Immune and Stress Responses in Eurasian Perch Perca fluviatilis Juveniles
}

\author{
Mahyar Zare, Hung Quang Tran (D), Marketa Prokešová and Vlastimil Stejskal *D \\ Faculty of Fisheries and Protection of Waters, South Bohemian Research Center of Aquaculture and Biodiversity \\ of Hydrocenoses, Institute of Aquaculture and Protection of Waters, University of South Bohemia in České \\ Budějovice, Husova třída 458/102, 37005 České Budějovice, Czech Republic; mzare@frov.jcu.cz (M.Z.); \\ htranquang@frov.jcu.cz (H.Q.T.); mprokesova@frov.jcu.cz (M.P.) \\ * Correspondence: stejskal@frov.jcu.cz; Tel.: +42-060-464-6621
}

check for updates

Citation: Zare, M.; Tran, H.Q.; Prokešová, M.; Stejskal, V. Effects of Garlic Allium satioum Powder on Nutrient Digestibility, Haematology, and Immune and Stress Responses in Eurasian Perch Perca fluviatilis Juveniles. Animals 2021, 11, 2735. https://doi.org/10.3390/ani11092735

Academic Editors: Fulvia Bovera and Giovanni Piccolo

Received: 9 August 2021

Accepted: 15 September 2021

Published: 19 September 2021

Publisher's Note: MDPI stays neutral with regard to jurisdictional claims in published maps and institutional affiliations.

Copyright: (c) 2021 by the authors. Licensee MDPI, Basel, Switzerland. This article is an open access article distributed under the terms and conditions of the Creative Commons Attribution (CC BY) license (https:/ / creativecommons.org/licenses/by/ $4.0 /)$.
Simple Summary: Herbal medicine feed supplements are used as growth promoters, immune system stimulants, and to combat stress. We evaluated the effects of garlic powder in the diet of European perch. The inclusion of garlic powder was shown to improve whole body composition, feed digestibility, and biochemical and immunohematological effects, and increased resistance against overcrowding stress.

Abstract: The supplementation of fish diets with phytogenics can increase growth performance and can modulate immune system response. European perch Perca fluviatilis (initial weight $25.0 \pm 0.4 \mathrm{~g}$ ) were fed a diet including 0 (Control), 10 (G10), 20 (G20), and 30 (G30) $\mathrm{g} \mathrm{kg}^{-1}$ garlic powder. No significant difference in the growth parameters and somatic indices were observed. Significantly higher fat digestibility was observed in G10 and G30 diets compared to in the control and G20 $\operatorname{diets}(p<0.05)$. Significantly greater red blood cell and white blood cell counts were observed with the G10 diet $(p<0.05)$. Garlic significantly decreased serum cholesterol in all of the experimental groups. Serum albumin was significantly higher in the G10 and G20 diets $(p<0.05)$. Immediately after the overcrowding stress challenge, the garlic groups showed significantly higher cortisol levels than the control group, while no significant difference was observed in the glucose concentration among groups. At $1 \mathrm{~h}$ post-stress, all of the groups that had been fed a garlic-supplemented diet showed lower cortisol levels than the control group, and this trend was maintained at 6 and $24 \mathrm{~h}$ post stress $(p<0.05)$, and glucose level in all garlic groups was significantly lower than control $(p<0.05)$. Garlic at $10 \mathrm{~g} \mathrm{~kg}^{-1}$ in feed can improve apparent fat digestibility and selected blood parameters and can enhance resistance against high-density and net handling stress in Eurasian perch.

Keywords: aquaculture; cortisol; fish; haematology; immunology; myeloid cells; stress

\section{Introduction}

Commercial production of fish, shellfish, and seafood is projected to increase by approximately $62 \%$ by 2030 [1]. Thus, the aquaculture rearing system is changing from an extensive system to intensive and semi-intensive systems [2], which might increase the chance of infectious disease outbreaks occurring [3]. Botanical derivatives and extracts, also known as phytogenics, have been used in fish diets as natural growth promoters and as immune stimulants. Currently, many plant extracts are considered as safe and cost-effective additives to aquafeed [4]. Antibiotics that can control pathogens on fish farms present concerns with respect to consumer health, animal welfare, and environmental pollution [5].

Garlic Allium satioum belongs to the Liliaceae family [6]. It has long been used as a herbal medicine and may be relevant to aquaculture because of its immunostimulant properties [7]. Garlic contains alliin, which has sulfur compounds including gamma- 
glutamyl-s-allyl-cysteine and S-allyl-L-cysteins sulfoxides. Moreover, garlic contains allicin (diallyl thiosulfate), which is responsible for garlic's typical pungent smell and its medicinal properties [6], and other bioactive compounds, including vitamins (ascorbic acid, thiamine and riboflavin), minerals (potassium, phosphorus, calcium, magnesium, sodium, iron, selenium, and germanium), flavonoids (phenolic acids) [8], and amino acids [9]. Garlic powder has been reported to promote growth in Japanese seabass (Lateolabrax japonicus) [10] and rainbow trout Oncorhynchus mykiss [11] and to improve body composition in brown trout (Salmo caspius) [12]. However, garlic powder was not shown to improve growth performance in Asian sea bass (Lates calarifer) [13] or rohu (Labeo rohita) [14]. In addition, it has been reported to increase apparent nutrient digestion in rainbow trout [15] and Nile tilapia (Oreochromis niloticus) [16]. Dietary garlic powder has shown a favourable effect on blood total protein, albumin, and phagocytic activity in rainbow trout $[17,18]$; this plant increased fish resistance to ammonia stress [19]. Garlic powder increased immunoglobulins in European seabass (Dicentrarchus labrax) [20]. Garlic microencapsulated extract improved growth performance, body proximate composition, and biochemical and immunohematological parameters in rainbow trout (Oncorhynchus mykiss) [21]. These studies, regardless of the form of garlic presentation within diets, suggest that garlic may be used as an alternative to antibiotics [22].

Eurasian perch (Perca fluviatilis) is a carnivorous percid fish inhabiting northern Eurasia [23]. It is a domesticated species with a wide habitat range, and can be found in brackish water, estuaries, and rivers in recent decades [24], showing potential for European inland culture [23]. Eurasian perch can be a suitable species for recirculation aquaculture system (RAS) production and intensive culture [25], but handling through counting, sorting, and tank cleaning as well as high density stocking, may potentially increase energy consumption and decrease feed intake and growth [26].

Regarding to use of Eurasian perch in RAS culture in recent decades [25], the aim of the present study was to investigate the effects of garlic powder in feed on growth performance, body proximate composition, apparent nutrient digestibility, selected blood and immune parameters, and resistance to high-density and net handling stress in Eurasian perch juveniles.

\section{Materials and Methods}

\subsection{Ethics Approval}

The experimental procedures were performed under guidelines of the European Communities Directive (No. 2010/63/EU) on the protection of animals used for scientific purposes and have been approved by the Czech Ministry of Health (MSMT-6744/2018-2).

\subsection{Preparation of Garlic Powder and Feed}

Garlic powder was purchased from EQUISERVIS, Prague, in Czech Republic. Garlic powder was produced by Pommier Nutrition, Thymerais-France. (Accessed: 4 June 2019) (www.pommier-nutrition.com). Experimental feeds (Table 1) were extruded at Exot Hobby s.r.o. (Černá v Pošumaví, Czech Republic), packed in plastic vacuum bags, and stored at $4{ }^{\circ} \mathrm{C}$ until use. In the present study, corn meal was replaced by 10, 20, and 30 g garlic powder per kilogram of diet feed. The proximate composition of the basal diet, including dry matter, crude protein, crude fat, and ash, was $93.48 \%, 47.20 \%, 16.33 \%$, and $8.91 \%$, respectively (Table 1 ).

\subsection{Experimental Design}

Eurasian perch juveniles with an initial weight of $25.0 \pm 0.4 \mathrm{~g}$ were obtained from Anapartners s.r.o fish farm (Prague, Czech Republic). Fish were transferred to the aquaria at the Institute of Aquaculture and Protection of Waters (České Budějovice, Czech Republic) and were fed a basal diet formulation (Table 1) without garlic powder for a 14-day acclimation period before the feeding trial [12]. After adaptation, 1320 fish were randomly distributed into twelve $150 \mathrm{~L}$ tanks (110 fish per tank) with a water flow rate $10 \mathrm{~L} \mathrm{~min}^{-1}$ in RAS [25]. 
Each diet was tested with three replicates. Fish were fed manually to apparent satiation at 08:00, 12:00, and 16:00 for 87 days, and uneaten feed was collected at maximum of $30 \mathrm{~min}$ after each meal. Water temperature, $\mathrm{pH}$, and dissolved oxygen (DO) were measured daily by an HQ40D portable multi-meter (Hach Lange $\mathrm{GmbH}$, Düsseldorf, Germany) and were maintained at $22.1 \pm 0.5^{\circ} \mathrm{C}$, $\mathrm{pH} 7.14 \pm 1.61$, and $\mathrm{DO} 8.16 \pm 0.42 \mathrm{mg} \mathrm{L}^{-1}$, respectively. The photoperiod was 12L:12D [27].

Table 1. Proximate composition of experimental diets with and without garlic powder.

\begin{tabular}{|c|c|c|c|c|}
\hline Ingredients $\left(\mathrm{g} \mathrm{kg}^{-1}\right)$ & Control & G10 & G20 & G30 \\
\hline Fish meal & 271 & 271 & 271 & 271 \\
\hline Soybean concentrate & 290 & 290 & 290 & 290 \\
\hline Corn meal & 97 & 87 & 77 & 67 \\
\hline Soybean meal & 128.5 & 128.5 & 128.5 & 128.5 \\
\hline Garlic powder ${ }^{a}$ & 0 & 10 & 20 & 30 \\
\hline Fish oil & 77 & 77 & 77 & 77 \\
\hline Rapeseed oil & 58 & 58 & 58 & 58 \\
\hline Methionine ${ }^{b}$ & 8 & 8 & 8 & 8 \\
\hline Lysine $^{c}$ & 5 & 5 & 5 & 5 \\
\hline Valine $^{\mathrm{d}}$ & 2 & 2 & 2 & 2 \\
\hline L-Threonine $\mathrm{e}^{\mathrm{e}}$ & 0.5 & 0.5 & 0.5 & 0.5 \\
\hline Vitamins \& minerals $\mathrm{f}$ & 8 & 8 & 8 & 8 \\
\hline Binder $\mathrm{g}$ & 50 & 50 & 50 & 50 \\
\hline Yttrium oxide $\left(\mathrm{Y}_{2} \mathrm{O}_{3}\right)^{\mathrm{h}}$ & 5 & 5 & 5 & 5 \\
\hline \multicolumn{5}{|c|}{ Proximate composition analysis } \\
\hline Dry matter\% & 93.48 & 93.54 & 93.71 & 93.17 \\
\hline Crude protein $\%$ & 47.20 & 46.84 & 46.59 & 46.33 \\
\hline Fat $\%$ & 16.33 & 16.51 & 15.98 & 16.13 \\
\hline Ash\% & 8.91 & 8.78 & 8.78 & 8.82 \\
\hline Fiber $\%$ & 1.82 & 3.87 & 3.98 & 2.42 \\
\hline Nitrogen-free extract (NFE) ${ }^{\mathrm{f}, \mathrm{i}}$ & 19.22 & 17.54 & 18.38 & 19.47 \\
\hline Gross energy $\left(\mathrm{Kj} \mathrm{g}^{-1}\right) \mathrm{g}, \mathrm{j}$ & 17.90 & 18.24 & 17.99 & 17.72 \\
\hline
\end{tabular}

${ }^{a}$ Garlic powder was purchased from EQUISERVIS, Prague, in Czech Republic. ${ }^{\mathrm{b}}$ Adisseo, Shanghai, China; ${ }^{\mathrm{c}}$ Inner Mongolia Eppen Biotech Co., Ltd., Ningxia, China.; ${ }^{\mathrm{d}}$ Ajinomoto Animal Nutrition Europe; ${ }^{\mathrm{e}}$ Ningxia Eppen Biotech, China; ${ }^{\mathrm{f}}$ Aminovitan Sak, Trouw Nutrition Biofaktory s.r.o, Prague, Czech Republic; ${ }^{g}$ binder (NutriBind, Adisseo, Shanghai, China) (3.0\%); ${ }^{\mathrm{h}}$ yttrium oxide $\left(\mathrm{Y}_{2} \mathrm{O}_{3}\right)$, Sigma, Ronkonkoma, NY, USA $;{ }^{i}$ nitrogen-free extracts (NFE) = dry matter-(crude protein + crude lipid + ash + fiber) [15]; ${ }^{j}$ gross energy was calculated according to following formula: gross energy $(\mathrm{MJ} / \mathrm{kg})=\left(\right.$ protein $\left.\times 23.6 \mathrm{~kJ} \mathrm{~g}^{-1}\right)+\left(\right.$ fat $\left.\times 39.5 \mathrm{~kJ} \mathrm{~g}^{-1}\right)+($ carbohydrates $\times$ $17.2 \mathrm{~kJ} \mathrm{~g}^{-1}$ ) [15]; control: without garlic supplement; G10: $10 \mathrm{~g}$ garlic powder per $1000 \mathrm{~g}$ diet; G20: $20 \mathrm{~g}$ garlic powder per $1000 \mathrm{~g}$ diet; G30: $30 \mathrm{~g}$ garlic powder per $1000 \mathrm{~g}$ diet.

\subsection{Growth Performance}

At the end of the feeding trial, feed was withheld for $24 \mathrm{~h}$. The fish were anesthetized by tricaine methane sulphonate (MS-222) at $200 \mathrm{mg} \mathrm{L}^{-1}$ water [21] and counted, and individual length and weight were measured. Growth performance and survival rate were calculated [21]. Final body weight (FBW), feed intake (FI), weight gain (WG), weight gain percentage (WG\%), feed conversion ratio (FCR), specific growth rate (SGR), protein efficiency ratio (PER), survival rate (SR\%), condition factor (CF\%), hepatosomatic index (HSI), and viscerosomatic index (VSI) were calculated as follows:

FBW $(\mathrm{g})=$ Final body weight

WG $(\mathrm{g})=$ [Final body weight $(\mathrm{g})$ - Initial body weight $(\mathrm{g})]$

$\mathrm{WG} \%=[$ Final body weight $(\mathrm{g})-$ Initial body weight $(\mathrm{g})] /$ initial body weight $(\mathrm{g}) \times 100$

FI $(\mathrm{g} /$ fish $)=$ dry feed consumed $/$ number of fish

PER $=$ Weight gain $(\mathrm{g}) /$ total protein intake $(\mathrm{g})$

$\mathrm{FCR}=$ dry feed intake $(\mathrm{g}) / \mathrm{WG}(\mathrm{g})$

SGR $\left(\%\right.$ day $\left.^{-1}\right)=100 \times[\ln$ final body weight $(\mathrm{g})-\ln$ initial body weight $(\mathrm{g})] /$ time (days)

$\mathrm{SR} \%=$ (number of fish after test $/$ initial number of stocked fish) $\times 100$

$\mathrm{CF} \%=\left[\right.$ fish body weight $\left.(\mathrm{g}) /(\text { fish length })^{3}(\mathrm{~cm})\right] \times 100$

$\mathrm{HSI} \%=[$ liver weight $(\mathrm{g}) /$ body weight $(\mathrm{g})] \times 100$ 
VSI $\%=[$ viscera weight $(\mathrm{g}) /$ body weight $(\mathrm{g})] \times 100$

\subsection{Whole Body Proximate Composition}

At the end of experiment, two fish per each tank ( $n=6$ per group) were randomly selected. Fish were anesthetized with MS-222 at $200 \mathrm{mg} \mathrm{L}^{-1}$ water [21] and were killed by a sharp blow to the head. The entire fish body was ground, packed individually into plastic bags, and stored at $-20^{\circ} \mathrm{C}$ for whole body proximate composition analysis. Both body and feed proximate composition analyses were conducted according to the methods of the Association of Official Analytical Chemists (AOAC) [28]. Crude fat was analyzed by the extraction method using hexane-isopropanol (3:2) according to Hara and Radin, [29] with slight modifications [30]. Crude protein was measured using the Kjeldahl method (BUCHI Labortechnik AG, type K-360, Königswinter, Germany) [12]. Dry matter was analyzed by drying in a NÜVE type FN 400P oven (NÜVE, Ankara, Turkey) at $105^{\circ} \mathrm{C}$ to a stable weight [21]. Ash was analyzed in a muffle furnace L 40/11 BO (Nabertherm $\mathrm{GmbH}$, Lilienthal, Germany)) at $550{ }^{\circ} \mathrm{C}$ for $4 \mathrm{~h} \mathrm{[12].}$

\subsection{Apparent Digestibility Coefficients}

Diets contained $5 \mathrm{~g} \mathrm{~kg}^{-1}$ yttrium oxide $\left(\mathrm{Y}_{2} \mathrm{O}_{3}\right)$ as an inert marker (Table 1). Two hours after the final daily meal, the tanks were brushed and cleaned, and the remaining faeces were discarded. After cleaning, the faeces were collected overnight by sieving every two hours from outlets until the first daily feeding in the morning. The faeces from each tank were stored separately at $-20{ }^{\circ} \mathrm{C}$ after centrifugation at $3000 \mathrm{rpm}$ for $15 \mathrm{~min}$ for analysis [31]. The $\mathrm{Y}_{2} \mathrm{O}_{3}$ in the diet and faeces was measured using inductively coupled plasma emission spectrometry following digestion with nitric acid at $180{ }^{\circ} \mathrm{C}$ for $48 \mathrm{~h}$. The apparent digestibility coefficients (ADC) of the dry matter, protein, and fat were calculated with the following formula [15]:

$\%$ digestibility $=100 \times 100[($ yttrium in feed $/$ yttrium in faeces $) \times($ nutrient in faeces $/$ nutrient in feed $)]$.

\subsection{Haematology and Biochemistry}

After $24 \mathrm{~h}$ starvation to induce the post-absorptive condition, two fish from each tank ( $n=6$ per group) were randomly netted and anesthetized by tricaine methane sulphonate (MS-222) at $200 \mathrm{mg} \mathrm{L}^{-1}$ water [21]. Duplicate blood samples were drawn from the caudal vein into heparinized and non-heparinized sterile syringes. The heparinized blood samples were transferred to heparinized Eppendorf tubes and were placed on ice for haematological analysis. The non-heparinized blood samples were transferred to non-heparinized Eppendorf tubes, and samples were left on ice for $2 \mathrm{~h}$ to clot [32]. The serum was subsequently separated by centrifugation (Heraeus Megafuge 16 R Centrifuge) at $4200 \mathrm{rpm}$ for $15 \mathrm{~min}$ at $4{ }^{\circ} \mathrm{C}$ and was stored at $-80^{\circ} \mathrm{C}$ until analysis [33]. Red blood cells (RBC), white blood cells (WBC), and subpopulations were counted according to a modified method of Korytár et al. [34]. The heparinized blood $(10 \mu \mathrm{L})$ was diluted in $200 \mu \mathrm{L}$ RPMI medium on ice for cell composition evaluation by a FACS Canto flow cytometer (Heidelberg, Germany). The biochemical parameters of the blood were assessed using an Abbott Architect c8000 clinical chemistry analyzer (Abbott, Chicago, IL, USA) and assay kits [19] according to manufacturer's instructions, as follows: serum total cholesterol, B7D6C7 G3-5321/R02 (Abbott, Chicago, IL, USA); triglycerides B7D7E7 G3-9334/R03 (Abbott, USA); alanine aminotransferase (ALT), B8L9x7 G5-4432/R05 (Abbott, USA); aspartate aminotransferase, G8-1502/R06 B8LY7 (Abbott, USA); albumin, 7D53-2030-3927/R6 (Abbott, USA); total protein, G6-6667/R04 B7D7D7 (Abbott, USA); and glucose, B3L8X7 G3-5375/R02) (Abbott, USA). Cortisol levels were analyzed with a cortisol assay kit (L2KCO2) using an immunochemistry analyzer Immulite 2000Xpi Siemens (Siemens Healthcare GmbH, Erlangen, Germany) at the Stafila laboratory, České Budějovice, Czech Republic. 


\subsection{Respiratory Burst and Phagocytic Activity}

Two fish per tank ( $n=6$ per group) were anesthetized with MS-222 (200 $\mathrm{mg} \mathrm{L}^{-1}$ water) [21]. The head kidney was removed, and the leukocytes were separated by pushing them through a nylon sieve with RPMI-1640 medium, according to the method by Biswas et al. [35]. A respiratory burst activity assay was conducted using nitro blue tetrazolium with minor modifications according to Zaineldin et al. [36]. Briefly, the leukocyte suspension was transferred into 96 -well plates, and an equivalent volume of $0.2 \%$ nitro blue tetrazolium solution (Sigma, Ronkonkoma, NY, USA) was added to each well and was incubated for $30 \mathrm{~min}$ at room temperature. After incubation, N-dimethylformamide (Sigma, USA) was added and centrifuged for $5 \mathrm{~min}$ at $3000 \mathrm{rpm}$. The respiratory burst activity was reported as the mean fluorescence intensity.

Phagocytic activity was assessed using a modified method from Morimoto et al. [37]. The leukocytes of head kidney were separated by washing with PBS $\left(5 \times 10^{5}\right.$ cells $\left.\mathrm{mL}^{-1}\right)$ and were incubated with latex beads at $25^{\circ} \mathrm{C}$ for $2 \mathrm{~h}$, after which cell-related fluorescence was evaluated, and the samples were transferred into 96-well plates and assessed with a FACS Canto flow cytometer (Heidelberg, Germany) to detect the fluorescence of the beads engulfed by the phagocytic cells.

\subsection{High-Density and Net Handling Stress Challenge}

At the end of the feeding period, working with one tank at a time, the volume of water was decreased to leave the fish in a high-density condition $(0.67 \mathrm{~kg} / \mathrm{L})$ for one minute with adequate aeration to avoid additional stress [38]. The fish were netted and removed from the water for $30 \mathrm{~s}$ [27] and were then returned to the tank, where the water level of the tank was increased back to the original volume, and the density was reduced [38]. Immediately after, two fish per tank were randomly selected ( $n=6$ per group), and the tank was refilled. The fish were anaesthetized with MS-222, and $1 \mathrm{~mL}$ of blood was drawn from the caudal vein with non-heparinized sterile syringes. All of these fish were killed after sampling. Sampling was conducted prior to the stress challenge, immediately after stress, and 1, 6 , and $24 \mathrm{~h}$ post-stress [27]. Blood sampling in each tank was completed within $5 \mathrm{~min}$.

\subsection{Statistical Analysis}

All statistical analyses were performed using IBM SPSS Statistics v. 22 (Armonk, NY, USA). Data normality and homogeneity were checked using the Kolmogorov-Smirnov test. Data were analyzed by one-way ANOVA. Significant differences among the mean values was set at $p<0.05$ using the Duncan test. In addition, to determine if the effect was linear and/or quadratic, a follow-up trend analysis using orthogonal polynomial contrasts was performed. The results are presented as mean $\pm \mathrm{SD}$ (standard deviation of the mean).

\section{Results}

\subsection{Growth Performance and Feed Utilization}

No significant differences in growth performance and feed utilization, including final weight, weight gain, weight gain percent, specific growth rate, feed intake, feed conversion ratio, protein efficiency ratio, or survival rate, were observed among the groups $(p>0.05)$. The condition factor was significantly lower in the G30 diet group compared to the other groups $(p<0.05)$. In particular, the condition factor significantly linearly decreased with the increasing dietary garlic powder levels $(p=0.04)$. In addition, no significant differences in the viscerosomatic or hepatosomatic index were found $(p>0.05)$. (Table 2). 
Table 2. Growth performance and feed utilization of juvenile Eurasian perch consuming feed supplemented with garlic powder.

\begin{tabular}{|c|c|c|c|c|c|c|c|c|c|c|}
\hline & & & & & & & $\begin{array}{l}\text { Linear } \\
\text { Trend }\end{array}$ & & $\begin{array}{l}\text { Quadratic } \\
\text { Trend }\end{array}$ & \\
\hline Parameters & Control & G10 & G20 & G30 & ANOVA & $\eta_{\mathrm{p}}^{2}$ & $P$-Value & $\mathbf{R}^{2}$ & $P$-Value & $\mathbf{R}^{2}$ \\
\hline Initial weight (g) & $24.75 \pm 0.47$ & $25.37 \pm 0.41$ & $24.77 \pm 0.13$ & $25.18 \pm 0.18$ & 0.13 & & & & & \\
\hline Final weight (g) & $66.29 \pm 1.99$ & $67.39 \pm 1.89$ & $64.89 \pm 1.53$ & $65.52 \pm 2.93$ & 0.55 & & & & & \\
\hline $\begin{array}{l}\text { Feed intake } \\
\left(\mathrm{g} \mathrm{fish}^{-1}\right)\end{array}$ & $68.49 \pm 1.82$ & $68.41 \pm 1.06$ & $68.00 \pm 0.80$ & $67.83 \pm 1.76$ & 0.92 & & & & & \\
\hline Weight gain $(\mathrm{g})$ & $41.53 \pm 2.02$ & $42.02 \pm 1.51$ & $40.12 \pm 1.40$ & $40.34 \pm 3.09$ & 0.65 & & & & & \\
\hline Weight gain $\%$ & $167.82 \pm 9.25$ & $165.55 \pm 3.67$ & $161.97 \pm 4.83$ & $160.29 \pm 13.38$ & 0.71 & & & & & \\
\hline $\begin{array}{c}\text { Feed conversion } \\
\text { ratio }\end{array}$ & $1.68 \pm 0.15$ & $1.64 \pm 0.07$ & $1.73 \pm 0.08$ & $1.71 \pm 0.16$ & 0.85 & & & & & \\
\hline $\begin{array}{l}\text { Specific growth } \\
\text { rate }\left(\% \text { day }^{-1}\right)\end{array}$ & $1.12 \pm 0.04$ & $1.11 \pm 0.01$ & $1.10 \pm 0.02$ & $1.09 \pm 0.05$ & 0.76 & & & & & \\
\hline $\begin{array}{c}\text { Protein efficiency } \\
\text { ratio }\end{array}$ & $1.25 \pm 0.09$ & $1.29 \pm 0.03$ & $1.24 \pm 0.07$ & $1.26 \pm 0.13$ & 0.90 & & & & & \\
\hline Survival rate $(\%)$ & $96.36 \pm 2.40$ & $98.18 \pm 0.91$ & $96.96 \pm 1.89$ & $97.57 \pm 2.78$ & 0.75 & & & & & \\
\hline Condition factor $\%$ & $1.25 \pm 0.05^{b}$ & $1.24 \pm 0.02^{b}$ & $1.18 \pm 0.03^{b}$ & $1.10 \pm 0.03^{a}$ & 0.00 & 0.78 & 0.04 & 0.33 & 0.05 & \\
\hline $\begin{array}{c}\text { Hepatosomatic } \\
\text { index } \%\end{array}$ & $1.59 \pm 0.36$ & $1.56 \pm 0.28$ & $1.53 \pm 0.37$ & $1.38 \pm 0.30$ & 0.44 & & & & & \\
\hline $\begin{array}{l}\text { Viscerosomatic } \\
\text { index } \%\end{array}$ & $12.06 \pm 2.28$ & $13.13 \pm 2.17$ & $12.88 \pm 1.56$ & $13.19 \pm 1.85$ & 0.49 & & & & & \\
\hline
\end{tabular}

Values are presented as (mean $\pm \mathrm{SD} ; n=110$ ). Mean values with different superscripts within a row vary significantly according to one-way ANOVA $(p<0.05) . \mathrm{R}^{2}=\mathrm{R}$ squared. $\eta_{\mathrm{p}}{ }^{2}=$ partial eta squared. Control: without garlic supplement; G10: $10 \mathrm{~g}$ garlic powder per $1000 \mathrm{~g}$ diet; G20: $20 \mathrm{~g}$ garlic powder per $1000 \mathrm{~g}$ diet; G30: $30 \mathrm{~g}$ garlic powder per $1000 \mathrm{~g}$ diet.

\subsection{Body Proximate Composition}

No significant differences were observed the among groups in terms of whole body dry matter, fat, or ash $(p>0.05)$. The level of protein in fish consuming the G30 diet was significantly higher than the G1 group $(p<0.05)$, but there were no significant differences among the controls, G10, and G20 groups ( $p>0.05)$ or among the controls, G20, and G30 groups $(p>0.05)$. There was a significant linear $(p=0.01)$ and quadratic $(p=0.04)$ trend regarding the dietary garlic powder level for body protein content, where body protein content decreased with the inclusion of garlic powder at G10 and then increased with the inclusion of garlic powder at G20 and G30. (Table 3).

Table 3. Body proximate composition of Eurasian perch consuming feed supplemented with garlic powder.

\begin{tabular}{|c|c|c|c|c|c|c|c|c|c|c|}
\hline & & & & & & & $\begin{array}{l}\text { Linear } \\
\text { Trend }\end{array}$ & & $\begin{array}{l}\text { Quadratic } \\
\text { Trend }\end{array}$ & \\
\hline Parameters & Control & G10 & G20 & G30 & ANOVA & $\eta_{\mathrm{p}}^{2}$ & $P$-Value & $\mathbf{R}^{2}$ & $P$-Value & $\mathbf{R}^{2}$ \\
\hline Dry matter\% & $32.08 \pm 1.15$ & $31.75 \pm 1.33$ & $32.10 \pm 1.71$ & $32.26 \pm 1.09$ & 0.92 & & & & & \\
\hline Fat $\%$ & $11.16 \pm 1.50$ & $10.96 \pm 1.44$ & $10.78 \pm 1.13$ & $9.76 \pm 1.24$ & 0.29 & & & & & \\
\hline Protein \% & $17.42 \pm 0.60^{\mathrm{ab}}$ & $16.98 \pm 0.69^{a}$ & $17.39 \pm 0.98^{a b}$ & $18.31 \pm 0.67^{b}$ & 0.04 & 0.33 & 0.01 & 0.23 & 0.04 & 0.25 \\
\hline Ash\% & $3.38 \pm 0.43$ & $3.01 \pm 0.60$ & $3.16 \pm 0.19$ & $3.53 \pm 0.29$ & 0.16 & & & & & \\
\hline
\end{tabular}

Values are presented as (mean $\pm \mathrm{SD} ; n=6$ ). Mean values with different superscripts within a row vary significantly according to one-way ANOVA $(p<0.05) . R^{2}=R$ square. $\eta_{\mathrm{p}}{ }^{2}=$ partial eta squared. Control: without garlic supplement; G10: $10 \mathrm{~g}$ garlic powder per $1000 \mathrm{~g}$ diet; G20: 20 g garlic powder per $1000 \mathrm{~g}$ diet; G30: $30 \mathrm{~g}$ garlic powder per $1000 \mathrm{~g}$ diet.

\subsection{Apparent Digestibility Coefficient (ADC\%)}

Significantly higher dry matter digestibility was observed in all of the garlic-supplemented groups compared to the controls $(p<0.05)$. Moreover, significantly higher fat digestibility was found in the G10 and G30 groups compared to the control and G20 groups $(p<0.05)$. No differences in protein digestibility were observed among the groups $(p>0.05)$. A positive linear $(p=0.00)$ and quadratic $(p=0.00)$ trend was found for dietary garlic powder levels and protein digestibility, where protein digestibility increased with the inclusion of garlic powder at G10 and then decreased with the inclusion of garlic powder at G20 and G30. (Table 4). 
Table 4. Apparent digestibility coefficient for dry matter, fat, and protein in Eurasian perch provided feed supplemented with garlic powder.

\begin{tabular}{|c|c|c|c|c|c|c|c|c|c|c|}
\hline & & & & & & & $\begin{array}{l}\text { Linear } \\
\text { Trend }\end{array}$ & & $\begin{array}{c}\text { Quadratic } \\
\text { Trend }\end{array}$ & \\
\hline Parameters & Control & G10 & G20 & G30 & ANOVA & $\eta_{\mathrm{p}}{ }^{2}$ & $P$-Value & $\mathbf{R}^{2}$ & $P$-Value & $\mathbf{R}^{2}$ \\
\hline $\mathrm{ADCd} \%$ & $77.53 \pm 0.59^{a}$ & $80.78 \pm 0.50^{b}$ & $79.60 \pm 0.87^{b}$ & $81.12 \pm 1.83^{b}$ & 0.01 & 0.71 & 0.69 & - & 0.27 & - \\
\hline $\mathrm{ADCf} \%$ & $78.29 \pm 0.46^{a}$ & $79.89 \pm 0.68^{b}$ & $78.35 \pm 0.72^{a}$ & $80.16 \pm 1.08^{b}$ & 0.03 & 0.65 & 0.94 & - & 0.09 & - \\
\hline $\mathrm{ADCp} \%$ & $92.41 \pm 0.30$ & $93.33 \pm 0.28$ & $92.66 \pm 0.32$ & $92.49 \pm 0.55$ & 0.06 & & & & & \\
\hline
\end{tabular}

Values are presented as (mean $\pm \mathrm{SD} ; n=6$ ). Mean values with different superscripts within a row vary significantly according to one-way ANOVA $(p<0.05) . \mathrm{R}^{2}=\mathrm{R}$ squared. $\eta_{\mathrm{p}}{ }^{2}=$ partial eta squared. Control: without garlic supplement; G10: $10 \mathrm{~g}$ garlic powder per $1000 \mathrm{~g}$ diet; G20: $20 \mathrm{~g}$ garlic powder per $1000 \mathrm{~g}$ diet; G30: $30 \mathrm{~g}$ garlic powder per $1000 \mathrm{~g}$ diet; ADCd: Apparent digestibility coefficient of dry matter. ADCf: Apparent digestibility coefficient of fat. ADCp: Apparent digestibility coefficient of protein.

\subsection{Haematology and Serum Biochemistry}

The number of RBCs and WBCs in G10 were significantly higher than those observed in the other groups $(p<0.05)$. The WBCs had positive quadratic trend $(p=0.01)$ to the dietary garlic powder and reached a peak in the G10 group. The RBCs had positive quadratic trend to the dietary G10 group ( $p=0.01)$. (Table 5).

Table 5. Haematological parameters of Eurasian perch fed with feeds supplemented with garlic powder.

\begin{tabular}{|c|c|c|c|c|c|c|c|c|c|c|}
\hline & & & & & & & $\begin{array}{l}\text { Linear } \\
\text { Trend }\end{array}$ & & $\begin{array}{l}\text { Quadratic } \\
\text { Trend }\end{array}$ & \\
\hline Parameters & Control & G10 & G20 & G30 & ANOVA & $\eta \mathrm{p}^{2}$ & $P$-Value & $\mathrm{R}^{2}$ & $P$-Value & $\mathrm{R}^{2}$ \\
\hline $\begin{array}{l}\text { Red blood cells } \\
\left(n \times 10^{6} \mu \mathrm{L}^{-1}\right)\end{array}$ & $283,896 \pm 77,236 \mathrm{ab}$ & $464,543 \pm 78,157^{c}$ & $256,285 \pm 16,266^{a}$ & $352,395 \pm 46,442 \mathrm{~b}$ & 0.00 & 0.68 & 0.13 & - & 0.01 & 0.34 \\
\hline $\begin{array}{l}\text { White blood cells } \\
\left(n \times 10^{6} \mu \mathrm{L}^{-1}\right)\end{array}$ & $19,711 \pm 5397$ & $30,589 \pm 7884 \mathrm{~b}$ & $18,520 \pm 4312^{\mathrm{a}}$ & $21,245 \pm 5152^{\mathrm{a}}$ & 0.00 & 0.44 & 0.03 & 0.19 & 0.01 & 0.33 \\
\hline $\begin{array}{l}\text { Lymphocytes }(\%) \\
\text { Myeloid cells } \% \text { ) }\end{array}$ & $\begin{array}{l}91.84 \pm 3.51 \\
8.15 \pm 3.51\end{array}$ & $\begin{array}{l}89.77 \pm 4.56 \\
10.22 \pm 4.56\end{array}$ & $\begin{array}{c}93.91 \pm 1.84 \\
6.08 \pm 1.84\end{array}$ & $\begin{array}{r}94.11 \pm 2.87 \\
5.88 \pm 2.87\end{array}$ & $\begin{array}{l}0.11 \\
0.11\end{array}$ & & & & & \\
\hline
\end{tabular}

Values are presented as (mean $\pm \mathrm{SD} ; n=6$ ). Mean values with different superscripts within a row vary significantly according to one-way ANOVA $(p<0.05) . R^{2}=R$ square. $\eta_{p}{ }^{2}=$ partial eta squared. Control: without garlic supplement; G10: $10 \mathrm{~g}$ garlic powder per $1000 \mathrm{~g}$ diet; G20: $20 \mathrm{~g}$ garlic powder per $1000 \mathrm{~g}$ diet; G30: $30 \mathrm{~g}$ garlic powder per $1000 \mathrm{~g}$ diet.

No significant differences in blood serum ALT and AST activity, triglycerides, or total protein were observed among the groups $(p>0.05)$. At all levels, garlic powder was associated with significantly lower levels of cholesterol $(p<0.05)$. Significantly higher levels of albumin were detected in the G10 and G20 groups compared to in the other groups $(p<0.05)$. A significant linear trend $(p=0.00)$ was observed regarding the dietary garlic powder level for albumin, where albumin increased with the inclusion of garlic powder at G10 and then decreased with the inclusion of garlic powder at G20 and G30. (Table 6).

Table 6. Serum biochemistry of Eurasian perch provided feed supplemented with garlic powder.

\begin{tabular}{|c|c|c|c|c|c|c|c|c|c|c|}
\hline & & & & & & & $\begin{array}{l}\text { Linear } \\
\text { Trend }\end{array}$ & & $\begin{array}{l}\text { Quadratic } \\
\text { Trend }\end{array}$ & \\
\hline Parameters & Control & G10 & G20 & G30 & ANOVA & $\eta_{\mathrm{p}}{ }^{2}$ & $P$-Value & $\mathbf{R}^{2}$ & $P$-Value & $\mathbf{R}^{2}$ \\
\hline $\begin{array}{c}\text { Alanine } \\
\text { aminotransferase } \\
\left(\text { ukat } \mathrm{L}^{-1}\right)\end{array}$ & $0.28 \pm 0.10$ & $0.27 \pm 0.07$ & $0.28 \pm 0.08$ & $0.19 \pm 0.09$ & 0.27 & & & & & \\
\hline $\begin{array}{l}\text { Aspartate } \\
\text { aminotransferase } \\
\left(\text { ukat } \mathrm{L}^{-1}\right)\end{array}$ & $1.39 \pm 0.89$ & $1.92 \pm 1.26$ & $1.83 \pm 1.42$ & $0.75 \pm 0.40$ & 0.24 & & & & & \\
\hline $\begin{array}{l}\text { Cholesterol } \\
\left(\mathrm{mmol} \mathrm{L}^{-1}\right)\end{array}$ & $8.28 \pm 1.43^{b}$ & $6.02 \pm 1.13^{a}$ & $7.01 \pm 0.61^{\mathrm{a}}$ & $6.27 \pm 0.44^{\mathrm{a}}$ & 0.00 & & & & & \\
\hline $\begin{array}{l}\text { Triglycerides } \\
\left(\mathrm{mmol} \mathrm{L}^{-1}\right)\end{array}$ & $9.28 \pm 1.94$ & $9.79 \pm 6.20$ & $14.59 \pm 4.41$ & $11.30 \pm 3.63$ & 0.17 & & & & & \\
\hline Albumin $\left(\mathrm{g} \mathrm{L}^{-1}\right)$ & $11.16 \pm 0.77^{b}$ & $13.10 \pm 1.28^{\mathrm{c}}$ & $12.83 \pm 1.47^{\mathrm{c}}$ & $9.53 \pm 1.28^{\mathrm{a}}$ & 0.00 & 0.61 & 0.00 & 0.55 & 0.00 & 0.55 \\
\hline $\begin{array}{l}\text { Total protein } \\
\quad\left(\mathrm{g} \mathrm{L}^{-1}\right)\end{array}$ & $43.18 \pm 3.52$ & $42.86 \pm 2.26$ & $43.46 \pm 1.94$ & $41.51 \pm 1.35$ & 0.52 & & & & & \\
\hline
\end{tabular}

Values are presented as (mean $\pm \mathrm{SD} ; n=6$ ). Mean values with different superscripts within a row vary significantly according to one-way ANOVA $(p<0.05) . \mathrm{R}^{2}=\mathrm{R}$ square. $\eta_{\mathrm{p}}{ }^{2}=$ partial eta squared. Control: without garlic supplement; G10: $10 \mathrm{~g}$ garlic powder per $1000 \mathrm{~g}$ diet; G20: 20 g garlic powder per $1000 \mathrm{~g}$ diet; G30: $30 \mathrm{~g}$ garlic powder per $1000 \mathrm{~g}$ diet. 


\subsection{Respiratory Burst and Phagocyte Activity}

Garlic powder inclusion did not affect respiratory burst activity $(p>0.05)$ or lymphocyte and myeloid cell phagocytic activity and index $(p>0.05)$ (Table 7).

Table 7. Immunological parameters of Eurasian perch provided feed supplemented with garlic powder.

\begin{tabular}{|c|c|c|c|c|c|c|c|c|c|c|}
\hline & & & & & & & $\begin{array}{l}\text { Linear } \\
\text { Trend }\end{array}$ & & $\begin{array}{c}\text { Quadratic } \\
\text { Trend }\end{array}$ & \\
\hline Parameters & Control & G10 & G20 & G30 & ANOVA & $\eta_{\mathrm{p}}^{2}$ & $P$-Value & $\mathbf{R}^{2}$ & $P$-Value & $\mathbf{R}^{2}$ \\
\hline $\begin{array}{c}\text { Respiratory } \\
\text { burst activity } \\
\text { (MFI) }\end{array}$ & $9290.33 \pm 1185.85$ & $7873.66 \pm 1267.97$ & $7688.00 \pm 1675.28$ & $10,838.00 \pm 4639.88$ & 0.60 & & & & & \\
\hline $\begin{array}{l}\text { Lymphocytes } \\
\text { phagocytic } \\
\text { activity\% }\end{array}$ & $42.10 \pm 8.51$ & $46.81 \pm 7.86$ & $40.25 \pm 10.62$ & $41.87 \pm 6.24$ & 0.57 & & & & & \\
\hline $\begin{array}{l}\text { Lymphocytes } \\
\text { phagocytic } \\
\text { index }\end{array}$ & $14.45 \pm 2.70$ & $14.43 \pm 4.86$ & $14.14 \pm 5.16$ & $14.47 \pm 5.25$ & 0.99 & & & & & \\
\hline $\begin{array}{l}\text { Myeloid } \\
\text { phagocytic } \\
\text { activity\% }\end{array}$ & $52.95 \pm 7.69$ & $48.03 \pm 8.25$ & $54.08 \pm 10.61$ & $52.20 \pm 6.78$ & 0.63 & & & & & \\
\hline $\begin{array}{l}\text { Myeloid } \\
\text { phagocytic } \\
\text { index }\end{array}$ & $16.15 \pm 2.50$ & $19.41 \pm 7.48$ & $17.37 \pm 7.43$ & $17.91 \pm 6.97$ & 0.85 & & & & & \\
\hline
\end{tabular}

Values are presented as (mean $\pm \mathrm{SD} ; n=6) . \mathrm{R}^{2}=\mathrm{R}$ square. $\eta_{\mathrm{p}}{ }^{2}=$ partial eta squared. Control: without garlic supplement; G10: $10 \mathrm{~g}$ garlic powder per $1000 \mathrm{~g}$ diet; G20: $20 \mathrm{~g}$ garlic powder per $1000 \mathrm{~g}$ diet; G30: $30 \mathrm{~g}$ garlic powder per $1000 \mathrm{~g}$ diet. MFI: Mean fluorescence intensity.

\subsection{High-Density and Net Handling Stress Challenge}

No significant differences in the level of serum cortisol and glucose were observed among the groups before stress $(p>0.05)$. Immediately after stress, all garlic diet groups showed significantly higher levels of cortisol compared to the control group $(p<0.05)$. No significant differences in glucose levels were observed among the groups $(p>0.05)$. At $1 \mathrm{~h}$, significantly higher cortisol was observed in the controls, and there was a significantly lower level in the G30 group compared to in the other groups $(p<0.05)$. At $1 \mathrm{~h}$, a positive quadratic trend was found between the increasing levels of garlic powder and serum cortisol $(p=0.00)$, where serum cortisol decreased with the inclusion of garlic powder at G10, increased with the inclusion of garlic powder at G20, and then decreased with the inclusion of garlic powder at G30. At $1 \mathrm{~h}$, the control and G10 groups showed significantly higher glucose compared to in the G20 and G30 groups, while the glucose level of the G20 group was significantly lower than that of the other groups $(p<0.05)$. At $6 \mathrm{~h}$, significantly higher and lower levels of cortisol were observed in the control and G20 groups, respectively, compared to in the other groups $(p<0.05)$, where the highest level of glucose was found in the control and G10 groups, and the lowest level of glucose was found in the G30 group at significant levels $(p<0.05)$. At $6 \mathrm{~h}$, a significant linear trend $(p=0.00)$ regarding the dietary garlic powder level was observed for serum glucose. With increasing levels of garlic powder, the serum glucose decreased linearly. A positive quadratic trend was observed between the increasing levels of garlic powder and the serum glucose, where the serum glucose increased with the inclusion of garlic powder at G10 and then decreased with the inclusion of garlic powder at G20 and G30. At $24 \mathrm{~h}$, significantly higher and lower cortisol levels were detected in the control and G30 groups, respectively $(p<0.05)$, while glucose was significantly higher in the control group than in the garlic-fed groups $(p<0.05)$ (Figures 1 and 2). 


\begin{tabular}{|c|c|c|c|c|c|c|c|c|c|c|c|c|c|c|}
\hline A & $\mathrm{L}$ & $\mathrm{Q}$ & A & $\mathrm{L}$ & Q & A & $\mathrm{L}$ & Q & A & $\mathrm{L}$ & Q & A & L & Q \\
\hline$P \underline{0.91}$ & 0.93 & 0.81 & 0.00 & 0.33 & 0.27 & 0.00 & 0.12 & 0.00 & 0.00 & 0.36 & 0.67 & 0.00 & 0.95 & 0.14 \\
\hline$R^{2}-$ & 0.00 & 0.02 & - & 0.04 & 0.11 & - & 0.10 & 0.48 & $\begin{array}{ll} \\
\end{array}$ & 0.03 & 0.03 & - & 0.00 & 0.16 \\
\hline$\eta p^{2} \quad 0.02$ & - & & 0.16 & - & - & 0.01 & -1 & & 0.10 & -1 & 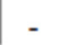 & 0.29 & - & 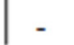 \\
\hline
\end{tabular}

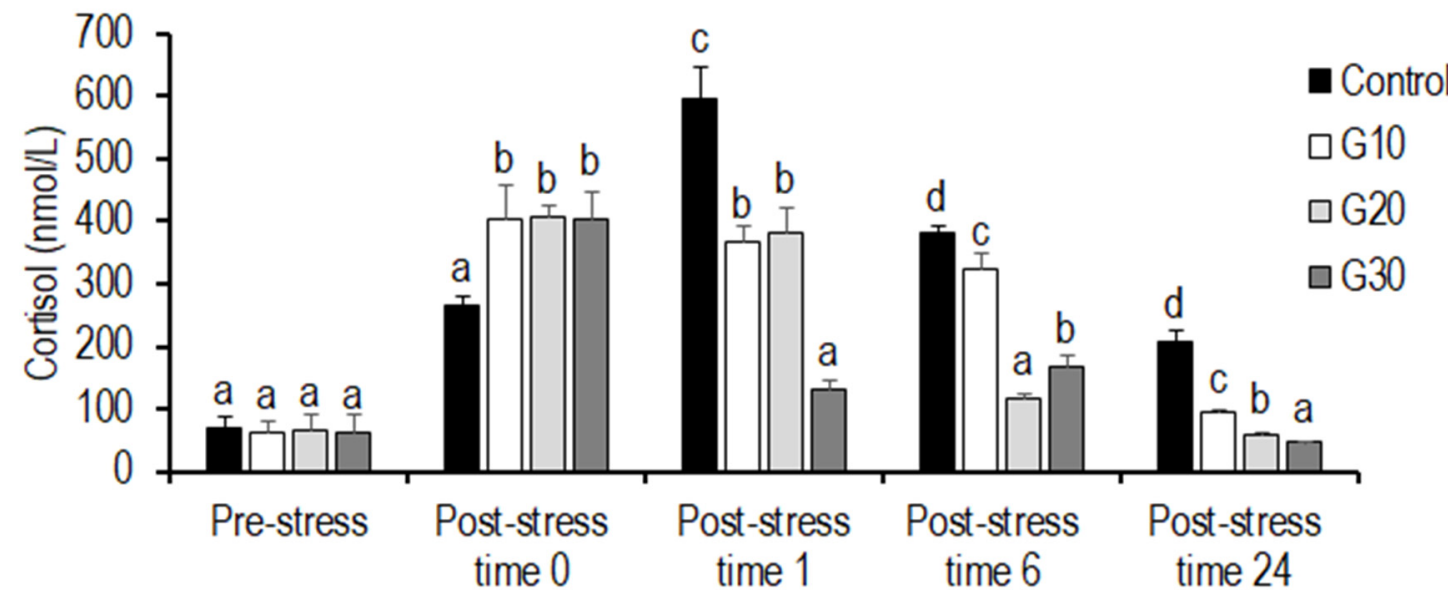

Figure 1. Serum cortisol of Eurasian perch provided feed supplemented with garlic powder under high-density and net handling stress. Values are presented as (mean $\pm \mathrm{SD} ; n=6$ ). Mean values with different superscripts within each time vary significantly according to one-way ANOVA $(p<0.05) . \mathrm{A}=$ the variance analysed by one-way ANOVA; $\mathrm{L}=$ the linear trend analysed by orthogonal polynomial contrasts; $\mathrm{Q}=$ the quadratic trend analysed by orthogonal polynomial contrasts. $\mathrm{R}^{2}=\mathrm{R}$ square. $\eta_{\mathrm{p}}{ }^{2}=$ partial eta squared. Control: without garlic supplement; G1: 10 g garlic powder per $1000 \mathrm{~g}$ diet; G2: $20 \mathrm{~g}$ garlic powder per $1000 \mathrm{~g}$ diet; G3: $30 \mathrm{~g}$ garlic powder per $1000 \mathrm{~g}$ diet. Pre-stress: before stress; Post-stress time 0: immediately after stress; Post-stress time 1: one hour after stress; Post-stress time 6: $6 \mathrm{~h}$ after stress; Post-stress time 24: $24 \mathrm{~h}$ after stress.

\begin{tabular}{|c|c|c|c|c|c|c|c|c|c|c|c|c|c|c|}
\hline A & L & $Q$ & A & $\mathrm{L}$ & $Q$ & A & L & $Q$ & A & $\mathrm{L}$ & $Q$ & A & $\mathrm{L}$ & $Q$ \\
\hline 0.63 & 0.76 & 0.93 & 0.00 & 0.39 & 0.99 & 0.00 & 0.13 & 0.15 & 0.00 & 0.00 & 0.00 & 0.00 & 0.09 & 0.17 \\
\hline - & 0.00 & 0.08 & & 0.00 & 0.00 & - & 0.10 & 0.1 & - & 0.45 & 0.46 & \begin{tabular}{l|}
- \\
\end{tabular} & 0.11 & 0.15 \\
\hline 20.1 & & & 0.01 & - & & 0.22 & 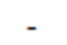 & & 0.31 & - & & 0.13 & - & \\
\hline
\end{tabular}

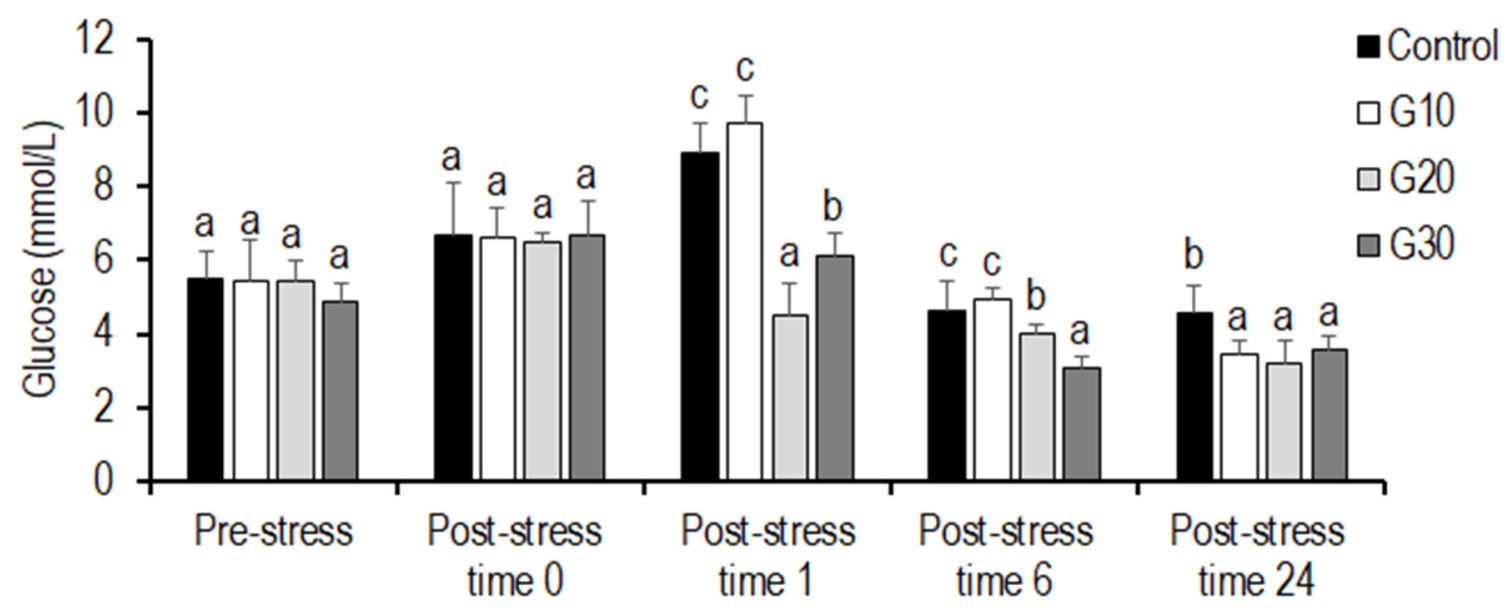

Figure 2. Serum glucose of Eurasian perch provided feed supplemented with garlic powder under high-density and net handling stress. Values are presented as (mean $\pm \mathrm{SD} ; n=6$ ). Mean values with different superscripts within each time vary significantly according to one-way ANOVA $(p<0.05) . \mathrm{A}=$ the variance analysed by one-way ANOVA; $\mathrm{L}=$ the linear trend analysed by orthogonal polynomial contrasts; $\mathrm{Q}=$ the quadratic trend analysed by orthogonal polynomial contrasts; $\mathrm{R}^{2}=\mathrm{R}$ square. $\eta_{\mathrm{p}}{ }^{2}=$ partial eta squared. Control: without garlic supplement; G1: $10 \mathrm{~g}$ garlic powder per $1000 \mathrm{~g}$ diet; G2: 20 g garlic powder per $1000 \mathrm{~g}$ diet; G3: $30 \mathrm{~g}$ garlic powder per $1000 \mathrm{~g}$ diet. Pre-stress: before stress; Post-stress time 0: immediately after stress; Post-stress time 1: one hour after stress; Post-stress time 6: $6 \mathrm{~h}$ after stress; Post-stress time 24: $24 \mathrm{~h}$ after stress. 


\section{Discussion}

In the present study, the inclusion of garlic powder in compound diets for European perch did not show significant effects on growth performance. This finding agrees with Sahu et al. [14], who reported that garlic powder in the diet of rohu at 1,5, and $10 \mathrm{~g} \mathrm{~kg}^{-1}$ feed did not significantly improve SGR or FCR. Another report documented that the use of garlic powder at the level of $40 \mathrm{~g} \mathrm{~kg}^{-1}$ in European sea bass did not have a significant effect on final weight, while $60 \mathrm{~g} \mathrm{~kg}^{-1}$ significantly decreased final weight, specific growth rate, and feed intake [39]. In contrast, garlic powder improved growth performance in Japanese sea bass at $25 \mathrm{~g} \mathrm{~kg}^{-1}$ [10], in brown trout at 20 and $30 \mathrm{~g} \mathrm{~kg}^{-1}$ [12], and in European sea bass at $10 \mathrm{~g} \mathrm{~kg}^{-1}$ [40]. Enhanced growth performance can be attributed to garlic bioactive compounds, including alliin, allicin, and organosulfur compounds, especially thiosulfinates [8], which increase digestion, nutrient uptake, and growth [16]. Differences among the results can be related to differences in the experimental design, fish species [10,12,40], fish size [39,40], garlic supplement type (powder or extract), and its purity $[41,42]$ and garlic supplement level in the diet [18,39].

The liver is active in fish metabolisms, and HSI can be a marker of the harmful effects from the environment or diet [43]. In our research, the HSI and VSI indices did not differ among groups. This agrees with Shalaby et al. [16], who reported no effect of garlic powder at 10, 20,30, and $40 \mathrm{~g} \mathrm{~kg}^{-1}$ feed on HSI in Nile tilapia. In contrast, $30 \mathrm{~g} \mathrm{~kg}^{1}$ garlic powder in the diet of brown trout [12] and $32 \mathrm{~g} \mathrm{~kg}^{-1}$ in the diet of Nile tilapia [42] were associated with significantly decreased HSI. In contrast, the inclusion of $10 \mathrm{~g} \mathrm{~kg}^{-1}$ garlic powder in the diet of brown trout also significantly increased HSI and VSI [12]. Furthermore, Lee et al. [44] confirmed that $5 \mathrm{~g} \mathrm{~kg}^{-1}$ of garlic extract did not have an effect on HSI in sterlet (Acipenser ruthenus) after 5 weeks, but $5 \mathrm{~g} \mathrm{~kg}^{-1}$ of garlic extract increased the somatic index (HSI) in sterlet after 10 weeks. Moreover, the use of garlic powder at levels 5, 10, 15, 20, and $30 \mathrm{~g} \mathrm{~kg}^{-1}$ in the sterlet diet significantly decreased HSI after a 12-week feeding trial in all garlic groups [45]. These reports showed that feeding trial duration has a strong effect on the hepatosomotic index. In contrast, our results confirm that no significant difference in HSI and VSI among groups can be significantly related to non-accumulation fat in the whole body and liver $[40,46]$ or reduced fat accumulation in the whole body and liver in the garlic groups [21,42].

The biological characteristics of fish along with environmental parameters, feeding protocols, and parasitic infections, affect the fish condition factor [47]. In recent studies, the addition of garlic powder to brown trout feed [12] did not increase the condition factor. In the present study, the condition factor in the G30 group was significantly reduced. Lower levels-10 $\mathrm{g} \mathrm{kg}^{-1}$ garlic powder in Japanese sea bass [10] and $20 \mathrm{~g} \mathrm{~kg}^{-1}$ in sterlet [45] feedsignificantly increased the condition factor, suggesting increased diet palatability [10,45]. In contrast, garlic powder at levels of 10,20 , and $30 \mathrm{~g} \mathrm{~kg}^{-1}$ significantly decreased the condition factor in Indian major carp, which is in line with our results [48]. In our study, the decrease in the condition factor can be attributed to the pungent odour of garlic in G30, which may have reduced feed palatability [49] and feed intake [39]. Moreover, previous reports proved that use of garlic powder in levels of $25 \mathrm{~g} \mathrm{~kg}^{-1}$ in the diet of Japanese seabass [10] and $60 \mathrm{~g} \mathrm{~kg}^{-1}$ [39] and $20 \mathrm{~g} \mathrm{~kg}^{-1}$ of garlic powder in European sea bass feed [40] decreased feed intake. In the present study, feed intake decreased in the G30 groups and subsequently decreased the condition factor [48] for Eurasian perch.

The whole-body proximate composition of perch fed garlic powder did not show significant differences in dry matter, fat, or ash, while the G30 diet significantly increased body proximate protein. These results are comparable to those with $30 \mathrm{~g} \mathrm{~kg}^{-1}$ garlic powder in brown trout [12] and $30 \mathrm{~g} \mathrm{~kg}^{-1}$ in monosex redbelly tilapia (Tilapia zilli) [50], which improved body proximate protein composition. The inclusion of garlic powder in the diet of European seabass [40] and Nile tilapia [16,42] improved body proximate composition. Studies have shown that garlic supplementation can increase body proximate protein. Increasing protein and decreasing fat can be attributed to the organosulfur compounds found in garlic such as allicin, S-allyl cysteine, and diallyl-di-sulfide, which reduce fat 
aggregation in the body [42] due to the increasing bile acids in the garlic treatments [51]. Bile acids are considered to be regulatory molecules, and they have been considered to stimulate specific nuclear receptors in cells in the liver and gastrointestinal tract [52]. Increased protein can be interpreted as a result of the essential amino acids contained in garlic [9], increasing free amino acids in the muscle and resulting in protein synthesis [40].

Plant ingredients in fish diets can balance some micronutrients or bioactive compounds [53]. The evaluation of the digestibility coefficients of feed ingredients specify the nutrient utilization for different fish species [54]. At our lowest test level, garlic powder significantly improved dry matter and fat digestibility. Esmaeili et al. [15] observed higher dry matter, fat, and protein digestibility in rainbow trout fed with $30 \mathrm{~g} \mathrm{~kg}^{-1}$ of garlic powder in feed. Shalaby et al. [16] demonstrated that $30 \mathrm{~g} \mathrm{~kg}^{-1}$ of garlic powder increased protein and fat digestibility in Nile tilapia, similar to our results in perch. Other studies have confirmed that garlic powder improved the digestibility of nutrients and SGR and decreased FCR in European seabass at 20 and $30 \mathrm{~g} \mathrm{~kg}^{-1}$ [40], in Nile tilapia at $32 \mathrm{~g} \mathrm{~kg}^{-1}$ [42], and in rainbow trout at $0.5,1,5$, and $10 \mathrm{~g} \mathrm{~kg}^{-1}$ [18]. Moreover, we found some studies showing that the use of $10 \mathrm{~g} \mathrm{~kg}^{-1}$ of garlic powder in the diet of sobaity sea bream (Sparidentex hasta) [55] and 5, 10, 15, and $20 \mathrm{~g} \mathrm{~kg}^{-1}$ of garlic powder in the diet of Asian sea bass significantly improved nutrient digestibility, SGR, and FCR [13]. Furthermore, the administration of microencapsulated garlic extract in rainbow trout at a level of $10 \mathrm{~g} \mathrm{~kg}^{-1}$ improved nutrient digestibility, SGR, and FCR as well [21]. These reports reveal that the administration of garlic as either a powder or an extract in different fish species increases growth performance [21,55] and nutrient digestibility due to the bioactive compounds found in garlic, such as allicin, which improved growth performance and nutrient digestibility in Nile tilapia [16,42] and European sea bass [40].

Red blood cell and withe blood cell counts are good key indices for evaluating fish physiology and pathology [56]. In our research, the administration of garlic at $10 \mathrm{~g} \mathrm{~kg}^{-1}$ increased RBC and WBC numbers compared to the other groups. Garlic powder has shown similar results in rainbow trout at $0.5,1,5$, and $10 \mathrm{~g} \mathrm{~kg}^{-1}$ [18] and in rohu at $10 \mathrm{~g} \mathrm{~kg}^{-1}$ [14]. Nya and Austin [18] reported that $10 \mathrm{~g} \mathrm{~kg}^{-1}$ of garlic powder increased the WBCs in rainbow trout but did not affect RBC numbers. In contrast, the administration of $10 \mathrm{~g} \mathrm{~kg}^{-1}$ of garlic extract (allicin) in the diet of rainbow trout increased RBC numbers, but significantly decreased WBCs [41]. The use of garlic powder did not alter RBC and WBC numbers in brown trout at 10, 20, or $30 \mathrm{~g} \mathrm{~kg}^{-1}$ [12] or in beluga (Huso huso) at $10 \mathrm{~g} \mathrm{~kg}^{-1}$ [57], and it had no effect on RBC numbers in European sea bass at 10, 20, or $30 \mathrm{~g} \mathrm{~kg}^{-1}$, while $30 \mathrm{~g} \mathrm{~kg}^{-1}$ of garlic powder increased the WBCs in sea bass [40]. The higher number of WBCs found in perch in our study may be related to the immunostimulatory effect of garlic compounds on the kidney, spleen, and thymus [58], as reported in previous studies $[13,18]$. RBCs play important roles in oxygen transfer, decreasing hypoxia stress, and contributing to fish health [59]. Our findings of higher RBC counts can be attributed to the effect of garlic compounds such as allicin [41] on the head kidney as the main erythropoietic site in teleost fish [60]. In our study, diets containing garlic powder did not increase concentrations of blood lymphocytes or myeloid cells. This result is similar to the inclusion of 5, 10, 15, and $20 \mathrm{~g} \mathrm{~kg}^{-1}$ in the diet of Asian sea bass [13]. Nya et al. [41] reported that $10 \mathrm{~g} \mathrm{~kg}^{-1}$ of allicin in the diet of rainbow trout increased neutrophil concentration but showed no effect on lymphocyte and monocyte percentage. WBCs, including lymphocytes [61] and myeloid cells [62], have key functions against pathogens as a first line of defence [63]. Myeloid cells include neutrophils and eosinophils (granulocytes) along with monocytes (macrophages) in fish [62].

Fish health can be evaluated by blood serum biochemical parameters [33], specifically the levels of ALT and AST [21,55], which are affected by diet, environment, and stress [64]. The level of ALT and AST activity is considered an indicator of liver health [33]. The levels of blood serum ALT and AST can be affected by stocking density [65]; water parameters [66]; and fish species [55,57], age, and sex [67]. In the present study, garlic powder did not show significant effects on serum ALT and AST activity. In agreement with our results, garlic 
powder in the $40 \mathrm{~g} \mathrm{~kg}^{-1}$ diet did not show significant effect on ALT and AST activity in Asian sea bass (Lates calcarifer) [68]. Furthermore, a mixture of cumin seeds (Nigella sativa) and turmeric (Curcuma longa Linn.) powder at the levels of 5 and $10 \mathrm{~g} \mathrm{~kg}^{-1}$ feed $(1: 1 \mathrm{w} / \mathrm{w})$ did not show significant difference in the levels of ALT and AST in the Asian sea bass (L. Calcarifer), which is the same as in our study [69]. Other studies showed no effect on ALT activity in sobaity sea bream [55] or beluga at $10 \mathrm{~g} \mathrm{~kg}^{-1}$ feed [57]. Serum AST activity significantly increased in sobaity sea bream with $10 \mathrm{~g} \mathrm{~kg}^{-1}$ of garlic [55] and decreased in beluga [57]. Garlic powder at $32 \mathrm{~g} \mathrm{~kg}^{-1}$ [42] and 30 and $40 \mathrm{~g} \mathrm{~kg}^{-1}$ significantly decreased blood serum ALT and AST activity in Nile tilapia [16]. Moreover, garlic powder at the levels 5, 10, and $15 \mathrm{~g} \mathrm{~kg}^{-1}$ in feed decreased the level of blood serum ALT and AST significantly in common carp (Cyprinus carpio) [70]. In contrast, the inclusion of 40 and $50 \mathrm{~g} \mathrm{~kg}^{-1}$ of garlic powder significantly increased blood serum ALT and AST activity in rainbow trout [33]. The present study showed that the levels of ALT and AST can at least be related to fish species and to herbal medicine level and species $[68,70]$ in the diet, similar to previous studies [55,69]. Moreover, no significant difference in the level of blood serum ALT and AST in our experimental fish, indicating that 10,20 , and $30 \mathrm{~g} \mathrm{~kg}^{-1}$ of garlic powder in perch diet were safe doses, as they did not disturb liver finction, as confirmed in the previous studies $[68,69]$. The reduction of ALT and AST activity in the blood serum can be attributed to the antioxidant compounds found in garlic, including S-allyl cysteine and diallyl-di-sulfide [71] and the flavonoids rutin, tangeretin, and nobiletin [72]. These antioxidant compounds hinder fat peroxidation in the cell membrane and prevent ALT and AST secretion into the blood [55].

Triglyceride and cholesterol were measured as blood serum biochemical parameters [55]. We observed no significant differences in the triglyceride levels among groups, while cholesterol was significantly lower in the garlic-fed groups. Garlic powder at 5, 10, 15 , and $20 \mathrm{~g} \mathrm{~kg}^{-1}$ feed reduced cholesterol and triglycerides in Asian sea bass [13] as well as in rainbow trout at 20,30, and $50 \mathrm{~g} \mathrm{~kg}^{-1}$ [33]. In contrast, $10 \mathrm{~g} \mathrm{~kg}^{-1}$ garlic powder in feed increased cholesterol and triglyceride levels in sobaity sea bream [5]. Apparently, garlic sulphur compounds reduce triglyceride levels in the blood serum [42]. Allicin is a main bioactive compound found in garlic that is responsible for hypolipidemia and hypocholesterolemia [73] and inhibits cholesterol biosynthesis [74]. In this line, our result showed that garlic powder at the higher level of G30 (30 g garlic powder per $\mathrm{kg}$ feed) significantly decreased blood serum cholesterol levels in our experimental species. In line with our study, Shalaby et al. [16] confirmed that garlic powder improved nutrient digestibility, SGR\%, and FCR and increased fat digestibilty. Moreover, garlic powder decreased whole body fat and blood plasma lipids in Nile tilapia (O. niloticus). In another research study that was of a similar design to ours, garlic powder improved SGR, FCR, and nutrient digestibility and decreased total blood serum lipids, triglycerides, and cholesterol in Asian sea bass [13]. Moreover, Adineh et al. [21] reported the use of microencapsulated garlic extract at the level of $10 \mathrm{~g} \mathrm{~kg}^{-1}$ feed in rainbow trout improved SGR\%, FCR, and nutrient digestibility and decreased whole body fat, which is the same as our results. Another study showed that garlic oil ( $0.15 \mathrm{~g} \mathrm{~kg}^{-1}$ feed) and powder $\left(32 \mathrm{~g} \mathrm{~kg}^{-1}\right.$ feed) increased nutrient digestibility by improving SGR\% and FCR and decreased fat accumulation in the whole body and in the blood serum triglycerides and cholesterol [42] like our study. Previous studies $[13,16,21,42]$ confirm our results and have demonstrated that whole body fat accumulation, apparent fat digestibility, and levels of blood serum triglycerides and cholesterol are related. In fact, those studies confirmed that increasing fat digestibility decreases fat accumulation in the whole body and reduces blood serum triglycerides and cholesterol [16,42].

In the present study, blood serum albumin was significantly higher in the G10 and G20 groups. Albumin has a protein structure. Albumin is primarily produced in the liver and prevents blood from leaking out of blood vessels. Albumin also transfers medicines and other substances across the blood for tissue growth and is used for tissue growth and healing [75]. Garlic powder increased blood serum albumin in amur carp [76] and rainbow 
trout [18]. The inclusion of garlic powder at levels of 10, 20, and $30 \mathrm{~g} \mathrm{~kg}^{-1}$ in brown trout feed did not significantly increase blood serum albumin [12], but an increase was seen in Asian sea bass at the levels of 5, 15, and $20 \mathrm{~g} \mathrm{~kg}^{-1}$ feed [13]. These differences in results can be related to the garlic dose and fish species as well as feed ingredient composition.

Blood serum protein parameters specifically show the status of fish as they react to internal and external factors [42]. Blood serum protein provides energy, creates new cells, reconstructs muscles, transports other nutrients such as messengers in the body, and supports the immune system [70]. We did not find blood serum total protein to differ among groups. This was also reported by Talpur and Ikhwanuddin [13], who administered garlic powder to Asian sea bass at the levels of 5, 10,15, $20 \mathrm{~g} \mathrm{~kg}^{-1}$ feed, and by Nya and Austin [17], who used 5 and $10 \mathrm{~g} \mathrm{~kg}^{-1}$ in the feed of rainbow trout. In contrast, garlic powder at $10 \mathrm{~g} \mathrm{~kg}^{-1}$ in the diet of sobaity sea bream [55] and at $20 \mathrm{~g} \mathrm{~kg}^{-1}$ in brown trout [12] increased blood serum total protein. Total protein indicates immune system status [77]. Increased blood serum protein in the garlic groups can be interpreted as a higher amount of amino acids in the garlic groups as well as higher amounts of sulfur compounds including S-allyl cysteine sulfoxide [9] and and stimulate liver to synthesize blood serum proteins [42].

Phytogenics enhance the immune system of fish [78], but in our study, garlic in the diet of perch did not improve respiratory burst activity. This finding is in agreement with Mahfouz et al. [79], who reported that $20 \mathrm{~g} \mathrm{~kg}^{-1}$ of garlic powder in Nile tilapia feed did not increase respiratory burst activity, which may be related to fish species, culture, and feeding conditions. Respiratory burst is a latent metabolic route in the cells and is activated upon pathogen exposure. It destroys pathogens through the synthesis of powerful oxidizing compounds [80]. The use of 5 and $10 \mathrm{~g} \mathrm{~kg}^{-1}$ of garlic powder in rainbow trout increased respiratory burst reactive oxygen species [17] and $15 \mathrm{~g} \mathrm{~kg}^{-1}$ in Amur carp (Cyprinus carpio haematopterus) diets [76] was shown to increase respiratory burst activity. Increasing superoxide anion production elevates reactive oxygen species [14]. The administration of $10 \mathrm{~g} \mathrm{~kg}^{-1}$ garlic powder to Asian sea bass [13] and 0.5 and $1 \mathrm{~g} \mathrm{~kg}^{-1}$ to rainbow trout [18] increased superoxide anion production $(p<0.05)$.

Phagocytic activity is considered to be an indicator of fish immune system activity [81]. We did not find the inclusion of garlic powder in the diet of Eurasian perch to be associated with the phagocytic activity of lymphocytes or myeloid cells, unlike another reports that indicate that garlic powder increased phagocytic activity and the phagocytic index in Nile tilapia at 10 and $20 \mathrm{~g} \mathrm{~kg}^{-1}$ [82], Asian sea bass at $20 \mathrm{~g} \mathrm{~kg}^{-1}$ [13], and rainbow trout at $10 \mathrm{~g} \mathrm{~kg}^{-1}$ [18]. Garlic extract (allicin) increased phagocytic activity in rainbow trout at 5 and $10 \mathrm{~g} \mathrm{~kg}^{-1}$ feed [41]. Fish species and the level of garlic can determine its effect on the immune system. The phagocytic boost of garlic powder or garlic extract $[18,41]$ can be attributed to the immunostimulatory effect of compounds such as allicin [41], germanium, and lectin [83]. However, the present study showed that garlic powder cannot boost phagocytic activity, at least in perch. Although we did not find a significant immune response in our experimental fish in our study, immune response may happen during a longer feeding trial, at higher levels of garlic powder [14], or with the use of garlic extract in the diet [41]. In light of this, Sahu et al. [14] mentioned that superoxide anion production, which elevates reactive oxygen species was significantly higher in garlic groups compared to in control groups after 20-, 40-, 60- and 70-day feeding trials. However, the level of superoxide anion production after 60 days was higher than it was at 20,40, and 70 days. This result shows that immune response can at the very least be related to feeding trial duration.

A mixture of 200 ppm garlic and labiatae essential oils (Delacon, Austria) (PHYTO diet) did not reduce blood plasma cortisol or glucose in European sea bass [84]. Garlic powder at 10, 20, and $30 \mathrm{~g} \mathrm{~kg}^{-1}$ feed in brown trout [12] and at 1, 5, and $10 \mathrm{~g} \mathrm{~kg}^{-1}$ in rohu [14] showed no significant effect on serum glucose, while it decreased levels of blood serum glucose at 5, 10, 15, and $20 \mathrm{~g} \mathrm{~kg}^{-1}$ in the feed of Asian sea bass [13] and $40 \mathrm{~g} \mathrm{~kg}^{-1}$ in Nile tilapia feed [16]. Zaefarian et al. [12] suggested that the efficacy of garlic supplementation 
intake can be related to culture conditions and fish species. The reduction of glucose in blood serum can be attributed to the effect of garlic organosulfur compounds such as alliin (S-allyl cysteine sulfoxide) [85] and diallyl trisulfide [86], which have been shown to stimulate insulin secretion in diabetic mice [85] and rats [86], respectively. Although increasing levels of amino acids elevate insulin secretion, especially in carnivorous fish [87], increasing blood glucose levels in fish also elevate insulin levels [88]. Garlic organosulfur compounds increase glycemic control through enhanced insulin secretion and increase insulin sensitivity [85].

Blood cortisol and glucose are considered primary and secondary stress indicators in fish [89]. Cortisol is the key circulating glucocorticoid in fish, and its level is indicated by its cytosolic receptor, which regulates the expression of genes involved in growth, metabolism, and immune function [90]. Cortisol, a common stress indicator increased blood glucose in response to stress [91].

In the present study, post-challenge, the observed blood serum cortisol was significantly higher in all of the garlic groups compared to the control group, while there was no difference in the serum levels $(p>0.05)$ among groups. Elevated blood serum glucose indicates a higher stress level, requiring fish to increase energy expenditure [92]. Along with serum cortisol, glucose increases in response to energy demands [93]. Under stress, catecholamines and cortisol exert an effect on hepatocytes and induce glycolysis and gluconeogenesis, leading to an increase serum glucose [94].

At $24 \mathrm{~h}$ post-stress, the G30 group showed lower blood serum cortisol and glucose compared to the other groups $(p>0.05)$. At 1,6 , and $24 \mathrm{~h}$ post-stress, blood serum cortisol was lower in all of the garlic groups compared to the levels in the control grpi. High-density stocking [95], handling [27], heat stress [96], and low water $\mathrm{pH}$ [66] have been reported to increase levels of cortisol and glucose in fish. The inclusion of $2 \mathrm{mg}$ nano selenium and $2 \mathrm{ppm}$ garlic extract reduced blood plasma cortisol and glucose in grass carp (Ctenopharyngodon idella) under stocking density stress [97], while 200 ppm of a mixture of garlic and labiatae essential oil (Delacon, Austria) (PHYTO diet) reduced blood serum cortisol after $2 \mathrm{~h}$ overcrowding stress but did not show any effect on blood glucose $(p>0.05)$ in European sea bass [84]. In the present study, lower cortisol and glucose may be attributed to the bioactive compounds found in garlic, including alliin and diallyl trisulfide [98], which were higher in the G30 diet compared to in the other diets [13,21,42].

\section{Conclusions}

Garlic powder at $10 \mathrm{~g} \mathrm{~kg}^{-1}$ diet shows beneficial effects on haematology, blood biochemical parameters, and the apparent digestibility of nutrients including fat. The inclusion of garlic at $30 \mathrm{~g} \mathrm{~kg}^{-1}$ improved whole-body protein composition and increased resistance against high-density and net handling stress in European perch.

Further research should include garlic A. sativum powder in the diets of European perch of different sizes and developmental stages to evaluate growth performance and haematological and immunological parameters, including digestive enzymes and liver antioxidant activity. We suggest further study to identify bioactive compounds in garlic that are effective in immune-related gene expression.

Author Contributions: Conceptualization, M.Z. and V.S.; methodology, M.Z. and H.Q.T.; data curation, M.Z.; formal analysis, M.Z., H.Q.T., and M.P.; funding acquisition, V.S., project administration, V.S., validation, M.Z. and V.S., writing — original draft preparation, M.Z. and V.S.; writing-review and editing, all the authors. All authors have read and agreed to the published version of the manuscript.

Funding: Study was financially supported by the Ministry of Agriculture of the Czech Republic by NAZV project (QK1810296).

Institutional Review Board Statement: The experimental procedures were performed under guidelines of the European Communities Directive (No. 2010/63/EU) on the protection of animals used for scientific purposes and have been approved by the Czech Ministry of Health (MSMT-6744/2018-2). 
Data Availability Statement: The data presented in this research is available on request from the corresponding author.

Acknowledgments: We thank to Tomáš Korytář, Pavel Šablatura, Jan Matoušek, Jan Dofek, and Marcellin Rutegwa, for their help with this study, which was financially supported by the Ministry of Agriculture of the Czech Republic by NAZV project (QK1810296).

Conflicts of Interest: The authors declare no conflict of interest.

\section{References}

1. Griffin, W.; Wang, W.; de Souza, M.C. The sustainable development goals and the economic contribution of fisheries and aquaculture. FAO Aquac. Newsl. 2019, 60, 51-52.

2. Dawood, M.A.; Koshio, S. Recent advances in the role of probiotics and prebiotics in carp aquaculture: A review. Aquaculture 2016, 454, 243-251. [CrossRef]

3. Yan, J.; Guo, C.; Dawood, M.A.O.; Gao, J. Effects of dietary chitosan on growth, lipid metabolism, immune response and antioxidant-related gene expression in Misgurnus anguillicaudatus. Benef. Microbes. 2017, 8, 439-449. [CrossRef]

4. Citarasu, T. Herbal biomedicines: A new opportunity for aquaculture industry. Aquac. Int. 2010, 18, 403-414. [CrossRef]

5. Jennings, S.; Stentiford, G.D.; Leocadio, A.M.; Jeffery, K.R.; Metcalfe, J.D.; Katsiadaki, J.D.I.; Auchterlonie, N.A.; Mangi, S.C.; Pinnegar, J.K.; Ellis, T.; et al. Aquatic food security: Insights into challenges and solutions from an analysis of interactions between fisheries, aquaculture, food safety, human health, fish and human welfare, economy and environment. Fish Fish. 2016, 17, 893-938. [CrossRef]

6. Santhosha, S.; Jamuna, P.; Prabhavathi, S.N. Bioactive components of garlic and their physiological role in health maintenance: A review. Food Biosci. 2013, 3, 59-74. [CrossRef]

7. Firmino, J.P.; Galindo-Villegas, J.; Reyes-Lo'pez, F.E.; Gisbert, E. Phytogenic bioactive compounds shape fish mucosal immunity. Front. Immunol. 2021, 12, 695973. [CrossRef]

8. Lawson, L.D. Garlic: A review of its medicinal effects and indicated active compounds. In Phytomedicines of Europe: Chemistry and Biological Activity; Lawson, L.S., Bauer, R., Eds.; American Chemical Society: Washington, DC, USA, 1998; pp. $176-209$.

9. Sasaki, J.; Lu, C.; Machiya, E.; Tanahashi, M.; Hamada, K. Processed black garlic (Allium sativum) extracts enhance anti-tumor potency against mouse tumors. Med. Aromat. Plant Sci. Biotechnol. 2007, 2, 278-281.

10. Xu, A.; Shang-Guan, J.; Li, Z.; Gao, Z.; Huang, Y.; Chen, Q. Effects of garlic powder on feeding attraction activity, growth and digestive enzyme activities of Japanese seabass, Lateolabrax japonicus. Aquac. Nutr. 2020, 26, 390-399. [CrossRef]

11. Büyükdeveci, M.E.; Balcázar, J.L.; Demirkale, I.; Suat Dikel, S. Effects of garlic-supplemented diet on growth performance and intestinal microbiota of rainbow trout (Oncorhynchus mykiss). Aquaculture 2018, 486, 170-174. [CrossRef]

12. Zaefarian, A.; Yeganeh, S.; Adhami, B. Dietary effects of garlic powder (Allium sativum) on growth, blood indices, carcass composition, and lysozyme activity in brown trout (Salmo caspius) and resistance against Yersinia ruckeri infection. Aquac. Int. 2017, 25, 1987-1996. [CrossRef]

13. Talpur, A.D.; Ikhwanuddin, M. Dietary effects of garlic (Allium sativum) on haemato-immunological parameters, survival, growth, and disease resistance against Vibrio harveyi infection in Asian sea bass, Lates calcarifer (Bloch). Aquaculture 2012, 364, 6-12. [CrossRef]

14. Sahu, S.; Das, B.K.; Mishra, B.K.; Pradhan, J.; Sarangi, N. Effect of Allium sativum on the immunity and survival of Labeo rohita infected with Aeromonas hydrophila. J. Appl. Ichthyol. 2007, 23, 80-86. [CrossRef]

15. Esmaeili, M.; Abedian Kenari, A.; Rombenso, A.N. Effects of fish meal replacement with meat and bone meal using garlic (Allium sativum) powder on growth, feeding, digestive enzymes and apparent digestibility of nutrients and fatty acids in juvenile rainbow trout (Oncorhynchus mykiss Walbaum, 1792). Aquac. Nutr. 2017, 23, 1225-1234. [CrossRef]

16. Shalaby, A.M.; Khattab, Y.A.; Abdel Rahman, A.M. Effects of garlic (Allium sativum) and chloramphenicol on growth performance, physiological parameters and survival of Nile tilapia (Oreochromis niloticus). J. Venom. Anim. Toxins Incl. Trop. Dis. 2006, 12, 172-201. [CrossRef]

17. Nya, E.J.; Austin, B. Development of immunity in rainbow trout (Oncorhynchus mykiss, Walbaum) to Aeromonas hydrophila after the dietary application of garlic. Fish Shellfish Immunol. 2011, 30, 845-850. [CrossRef]

18. Nya, E.J.; Austin, B. Use of garlic, Allium sativum, to control control Aeromonas hydrophila infections in rainbow trout, Oncorhynchus mykiss (Walbaum). J. Fish Dis. 2009, 32, 963-970. [CrossRef] [PubMed]

19. Esmaeili, M.; Abedian Kenari, A.; Rombenso, A. Immunohematological status under acute ammonia stress of juvenile rainbow trout (Oncorhynchus mykiss Walbaum, 1792) fed garlic (Allium sativum) powder-supplemented meat and bone meal-based feeds. Comp. Clin. Path. 2017, 26, 853-866. [CrossRef]

20. Abdel-Tawwab, M.; Khalil, R.H.; Diab, A.M.; Khallaf, M.A.; Abdel-Razek, N.; Abdel-Latif, H.M.; Khalifa, E. Dietary garlic and chitosan enhance the antioxidant capacity, immunity, and modulate the transcription of HSP70 and Cytokine genes in Zearalenone-intoxicated European seabass. Fish Shellfish Immunol. 2021, 113, 35-41. [CrossRef]

21. Adineh, H.; Harsij, M.; Jafaryan, H.; Asadi, M. The effects of microencapsulated garlic (Allium sativum) extract on growth performance, body composition, immune response and antioxidant status of rainbow trout (Oncorhynchus mykiss) juveniles. J. Appl. Anim. Res. 2020, 48, 372-378. [CrossRef] 
22. Lee, J.Y.; Gao, Y. Review of the Application of Garlic, Allium sativum, in Aquaculture. J. World Aquac. Soc. 2012, 43, 447-458. [CrossRef]

23. Policar, T.; Samarin, A.M.; Mélard, C. Culture methods of Eurasian perch during on growing. In Biology and Culture of Percid Fishes: Principles and Practices; Kestemont, P., Dabrowski, K., Summerfelt, R.C., Eds.; Springer: Dordrecht, The Netherlands, 2015; pp. 417-435.

24. Ozerov, M.Y.; Ahmad, F.; Gross, R.; Pukk, L.; Kahar, S.; Kisand, V.; Vasemägi, A. Highly continuous genome assembly of Eurasian perch (Perca fluviatilis) using linked-read sequencing. G3 2018, 8, 3737-3743. [CrossRef]

25. Stejskal, V.; Kouril, J.; Valentova, O.; Hamackova, J.; Policar, T. Size-related oxygen consumption and ammonia excretion of Eurasian perch (Perca fluviatilis L.) reared in a recirculating system. Aquac. Res. 2009, 41, 135-142. [CrossRef]

26. Strand, A.; Magnhagen, C.; Alanärä, A. Effects of repeated disturbances on feed intake, growth rates and energy expenditures of juvenile perch, Perca fluviatilis. Aquaculture 2007, 265, 163-168. [CrossRef]

27. Acerete, L.; Balasch, J.C.; Espinosa, E.; Josa, A.; Tort, L. Physiological responses in Eurasian perch (Perca fluviatilis, L.) subjected to stress by transport and handling. Aquaculture 2004, 237, 167-178. [CrossRef]

28. Association of Official Analytical Chemists (AOAC). Official Methods of Analysis, 16th ed.; Association of Official Analytical Chemists: Washington, DC, USA, 2005.

29. Hara, A.; Radin, N.S. Lipid extraction of tissues with a low toxicity solvent. Anal. Biochem. 1987, 90, 420-426. [CrossRef]

30. Zajic, T.; Mraz, J.; Samples, S.; Pickova, J. Fillet quality changes as a result of purging of common carp (Cyprinus carpio L.) with special regard to weight loss and lipid profile. Aquaculture 2013, 400, 111-119. [CrossRef]

31. Vandenberg, G.W.; De La Noüe, J. Apparent digestibility comparison in rainbow trout (Oncorhynchus mykiss) assessed using three methods of faeces collection and three digestibility markers. Aquac. Nutr. 2001, 7, 237-245. [CrossRef]

32. Awad, E.; Austin, D.; Lyndon, A.R. Effect of black cumin seed oil (Nigella sativa) and nettle extract (Quercetin) on enhancement of immunity in rainbow trout, Oncorhynchus mykiss (Walbaum). Aquaculture 2013, 388, 193-197. [CrossRef]

33. Mohebbi, A.; Nematollahi, A.; Dorcheh, E.E.; Asad, F.G. Influence of dietary garlic (Allium sativum) on the antioxidative status of rainbow trout (Oncorhynchus mykiss). Aquac. Res. 2012, 43, 1184-1193. [CrossRef]

34. Korytář, T.; Thi, H.D.; Takizawa, F.; Köllner, B. A multicolour flow cytometry identifying defined leukocyte subsets of rainbow trout (Oncorhynchus mykiss). Fish Shellfish Immunol. 2013, 35, 2017-2019. [CrossRef]

35. Biswas, G.; Korenaga, H.; Nagamine, R.; Takayama, H.; Kawahara, S.; Takeda, S.; Kikuchi, Y.; Dashnyam, B.; Kono, T.; Sakai, M. Cytokine responses in the Japanese pufferfish (Takifugu rubripes) head kidney cells induced with heat-killed probiotics isolated from the Mongolian dairy products. Fish Shellfish Immunol. 2013, 34, 1170-1177. [CrossRef] [PubMed]

36. Zaineldin, A.I.; Hegazi, S.; Koshio, S.; Ishikawa, M.; Bakr, A.; El-Keredy, A.M.S.; Dawood, M.A.O.; Dossou, S.; Wang, W.; Yukuna, Z. Bacillus subtilis as probiotic candidate for red sea bream: Growth performance, oxidative status, and immune response traits. Fish Shellfish Immunol. 2018, 79, 303-312. [CrossRef] [PubMed]

37. Morimoto, T.; Biswas, G.; Kono, T.; Sakai, M.; Hikima, J. Immune responses in the Japanese pufferfish (Takifugu rubripes) head kidney cells stimulated with particulate silica. Fish Shellfish Immunol. 2016, 49, 84-90. [CrossRef] [PubMed]

38. Di Marco, P.; Priori, A.; Finoia, M.G.; Massari, A.; Mandich, A.; Marino, G. Physiological responses of European sea bass Dicentrarchus labrax to different stocking densities and acute stress challenge. Aquaculture 2008, 275, 319-328. [CrossRef]

39. İrkin, L.C.; Yiğit, M. The use of garlic (Allium sativum) meal as a natural feed supplement in diets for European seabass (Dicentrarchus labrax) juveniles. J. Aquac. Eng. Fish. Res. 2015, 2, 128-141. [CrossRef]

40. Saleh, N.E.; Michael, F.R.; Toutou, M.M. Evaluation of garlic and onion powder as phyto-additives in the diet of sea bass (Dicentrarcus labrax). Egypt. J. Aquat. Res. 2015, 41, 211-217. [CrossRef]

41. Nya, E.J.; Dawood, Z.; Austin, B. The garlic component, allicin, prevents disease caused by Aeromonas hydrophila in rainbow trout, Oncorhynchus mykiss (Walbaum). J. Fish Dis. 2010, 33, 293-300. [CrossRef]

42. Metwally, M.A.A. Effects of Garlic (Allium sativum) on Some Antioxidant Activities in Tilapia Nilotica (Oreochromis niloticus). World J. Fish Mar. Sci. 2009, 1, 56-64.

43. Pait, A.S.; Nelson, J.O. Vitellogenesis in male Fundulus heteroclitus (Killifish) induced by selected estrogenic compounds. Aquat. Toxicol. 2003, 64, 331-342. [CrossRef]

44. Lee, D.-H.; Ra, C.-S.; Song, Y.-H.; Sung, K.-I.; Kim, J.-D. Effects of dietary garlic extract on growth, feed utilization and whole body composition of juvenile sterlet sturgeon (Acipenser ruthenus). Asian-australas. J. Anim. Sci. 2012, 25, 577-583. [CrossRef] [PubMed]

45. Lee, D.H.; Lim, S.R.; Han, J.J.; Lee, S.W.; Ra, C.S.; Kim, J.D. Effects of dietary garlic powder on growth, feed utilization and whole-body composition changes in fingerling Sterlet Sturgeon, Acipenser ruthenus. Asian-australas. J. Anim. Sci. 2014, 25, 577-583. [CrossRef]

46. Hwang, J.-H.; Lee, S.-W.; Rha, S.-J.; Yoon, H.-S.; Park, E.-S.; Han, K.-H.; Kim, S.-J. Dietary green tea extract improves growth performance, body composition, and stress recovery in the juvenile black rockfish, Sebastes schlegeli. Aquac. Int. 2013, 21, 525-538. [CrossRef]

47. Froese, R.; Tsikliras, A.C.; Stergiou, K.I. Editorial note on weight-length relations of fishes. Acta Ichthyol. Piscat. 2011, 41, 261-263. [CrossRef]

48. Kaur, H.; Ansal, M.D. Efficacy of garlic powder as a growth promoting feed additive for higher growth and improved flesh quality in an Indian major carp, Labeo rohita (Ham.) fingerlings. J. Entomol. 2020, 8, 25-29.

49. Platel, K.; Srinivasan, K. Digestive stimulant action of spices: A myth or reality? Indian J. Med. Res. 2004, 119, 167-179. 
50. Ajiboye, O.O.; Yakubu, A.F.; Simpa, J.O.; Balogun, S.A. Effect of garlic-supplemented diets on growth response, survival, nutrient utilization and body composition of monosex Tilapia zillii. World J. Fish Mar. Sci. 2016, 8, 115-122.

51. Platel, K.; Srinivasan, K. Stimulatory influence of select spices on bile secretion in rats. Nutr. Res. 2000, 20, 1493-1503. [CrossRef]

52. Hylemon, P.B.; Zhou, H.; Pandak, W.M.; Ren, S.; Gil, G.; Dent, P. Bile acids as regulatory molecules. J. Lipid Res. 2009, 50, 1509-1520. [CrossRef]

53. Barrows, F.T.; Gaylord, T.G.; Sealey, W.M.; Smith, C.E.; Porter, L. Supplementation of plant-based diets for rainbow trout (Oncorhynchus mykiss) with macro-minerals and inositol. Aquac. Nutr. 2010, 16, 654-661. [CrossRef]

54. Lee, S.-M. Apparent digestibility coefficients of various feed ingredients for juvenile and grower rockfish (Sebastes schlegeli). Aquaculture 2002, 207, 79-95. [CrossRef]

55. Jahanjoo, V.; Yahyavi, M.; Akrami, R.; Bahri, A.H. Influence of adding garlic (Allium sativum), ginger (Zingiber officinale), thyme (Thymus vulgaris) and their combination on the growth performance, haemato-immunological parameters and disease resistance to Photobacterium damselae in sobaity sea bream (Sparidentex hasta) fry. Turkish J. Fish. Aquat. Sci. 2018, 18, $633-645$.

56. Fazio, F. Fish haematology analysis as an important tool of aquaculture: A review. Aquaculture 2019, 500, 237-242. [CrossRef]

57. Kanani, H.G.; Nobahar, Z.; Kakoolaki, S.; Jafarian, H. Effect of ginger- and garlic-supplemented diet on growth performance, some hematological parameters and immune responses in juvenile Huso huso. Fish Physiol. Biochem. 2014, 40, 481-490. [CrossRef] [PubMed]

58. Press, C.M.; Evensen, Ø. The morphology of the immune system in teleost fishes. Fish Shellfish Immunol. 1999, 9, 309-318. [CrossRef]

59. Mbokane, E.M.; Moyo, N.A.G. Effect of dietary Artemisia afra on growth, some innateimmunological parameters in Clarias gariepinus challenged with Aeromonas hydrophila. Aquac. Int. 2020, 28, 539-553. [CrossRef]

60. Witeska, M. Erythrocytes in teleost fishes: A review. Zool Ecol. 2013, 23, 275-281. [CrossRef]

61. Scapigliati, G. Functional aspects of fish lymphocytes. Dev. Comp. Immunol. 2013, 41, 200-208. [CrossRef] [PubMed]

62. Crowhurst, M.O.; Layton, J.E.; Lieschke, G.J. Developmental biology of zebrafish myeloid cells. Int. J. Dev. Biol. 2002, 46, 483-492.

63. Lieschke, G.; Trede, N. Fish immunology. Curr. Biol. 2009, 19, R678-R682. [CrossRef]

64. Barcellos, L.J.G.; Kreutz, L.C.; Souza, C.; Rodriguez, L.B.; Fioreze, I.; Quevedo, R.M.; Cericato, L.; Soso, A.B.; Fagundes, M.; Conrad, J.; et al. Haematological changes in jundia (Rhamdia quelen) after acute and chronic stress caused by usual aquacultural management, with emphasis on immunosuppressive effects. Aquaculture 2004, 237, 229-236. [CrossRef]

65. Battisti, E.K.; Rabaioli, A.; Uczay, J.; Sutili, F.J.; Lazzari, R. Effect of stocking density on growth, hematological and biochemical parameters and antioxidant status of silver catfish (Rhamdia quelen) cultured in a biofloc system. Aquaculture 2020, 524, 735213. [CrossRef]

66. Chan, T.L.; Roy, S.D.; Sharma, A.; Biswas, P.; Siddhya, G.M.; Das, A. Antistress potential of acetone extract of Zingiber officinale Roscoe on biochemical and oxidative stress parameters in Labeo calbasu (Hamilton, 1822) fingerlings subjected to acid stress. Indian J. Fish. 2014, 61, 69-77.

67. Akrami, R.; Gharaei, A.; Karami, R. Age and sex specific variation in hematological and serum biochemical parameters of Beluga (Huso huso Linnaeus, 1758). Int. J. Aquat. Biol. 2013, 1, 132-137.

68. Abdelwahab, A.M.; El-Bahr, S.M.; Al-Khamees, S. Influence of dietary garlic (Allium sativum) and/or ascorbic acid on performance, feed utilization, body composition and hemato-biochemical parameters of juvenile Asian Sea Bass (Lates calcarifer). Animals 2020, 10, 2396. [CrossRef]

69. Abdelwahab, A.; El-Bahr, S. Influence of black cumin seeds (Nigella sativa) and turmeric (Curcuma longa Linn.) mixture on performance and serum biochemistry of Asian sea bass, Lates calcarifer. World J. Fish Mar. Sci. 2012, 4, 496-503.

70. Yousefi, M.; Vatnikov, Y.A.; Kulikov, E.V.; Plushikov, V.G.; Drukovsky, S.G.; Hoseinifar, S.H.; Van Doan, H. The protective effects of dietary garlic on common carp (Cyprinus carpio) exposed to ambient ammonia toxicity. Aquaculture 2020, 526, 735400. [CrossRef]

71. Amagase, H.; Petesch, B.L.; Matsuura, H.; Kasuga, S.; Itakura, Y. Recent advances on the nutritional effects associated with the use of garlic as a supplement intake of garlic and its bioactive components. J. Nutr. 2001, 131, 955S-962S. [CrossRef] [PubMed]

72. Berginc, K.; Milisav, I.; Kristl, A. Garlic flavonoids and organosulfur compounds: The impact on saqunavir's and darunavir's hepatic pharmacokinetics. Drug Metab. 2010, 25, 521-530. [CrossRef] [PubMed]

73. Liu, L.; Yeh, Y.-Y. S-alk (en) yl cysteines of garlic inhibit cholesterol synthesis by deactivating HMG-CoA reductase in cultured rat hepatocytes. J. Nutr. 2002, 132, 1129-1134. [CrossRef] [PubMed]

74. Yamamoto, Y.; Oue, E. Antihypertensive effect of quercetin in rats fed with a high-fat high-sucrose diet. Biosci. Biotechnol. Biochem. 2006, 70, 933-939. [CrossRef]

75. Dhinaa, A.N.; Palanisamy, P.K. Z-Scan technique: To measure the total protein and albumin in blood. J. Biomed. Eng. 2010, 3, 285-290. [CrossRef]

76. Chesti, A.; Chauhan, R.S.; Khati, A. Evaluation of immunostimulatory effect of dietary garlic (Allium sativum) in fingerlings of Amur carp, (Cyprinus carpio haematopterus). J. Pharm. Innov. 2018, 7, 375-379.

77. Jha, A.K.; Pal, A.K.; Sahu, N.P.; Kumar, S.; Mukherjee, S.C. Haemato-immunological responses to dietary yeast RNA, w-3fatty acid and beta-carotene in Catla catla juveniles. Fish Shellfish Immunol. 2007, 23, 917-927. [CrossRef] [PubMed]

78. Van Hai, N. The use of medicinal plants as immunostimulants in aquaculture: A review. Aquaculture 2015, 446, 88-96. [CrossRef]

79. Mahfouz, N.B.; Fathi, M.; Zead, M.Y.A.; Mesalhy, S. Effect of garlic and yeast in the culture of Nile tilapia (Oreochromis niloticus). Abbassa Int. J. Aquac. 2009, 4, 453-465. 
80. Dale, D.C.; Boxer, L.; Liles, W.C. The phagocytes: Neutrophils and monocytes. Blood 2008, 112, 935-945. [CrossRef] [PubMed]

81. Abarike, E.D.; Kuebutornye, F.K.; Jian, J.; Tang, J.; Lu, Y.; Cai, J. Influences of immunostimulants on phagocytes in cultured fish: A mini review. Rev. Aquac. 2019, 11, 1219-1227. [CrossRef]

82. Diab, A.M.; Saker, O.; Eldakroury, M.; Elseify, M. Effects of garlic (Allium sativum) and curcumin (Turmeric, Curcuma longa Linn) on Nile tilapia immunity. Vet. Med. J.-Giza 2014, 60, 1-19.

83. Magnadóttir, B. Innate immunity of fish (overview). Fish Shellfish Immunol. 2006, 20, 137-151. [CrossRef]

84. Serradell, A.; Torrecillas, S.; Makol, A.; Valdenegro, V.; Fernández-Montero, A.; Acosta, F.; Izquierdo, M.S.; Montero, D. Prebiotics and phytogenics functional additives in low fish meal and fish oil based diets for European sea bass (Dicentrarchus labrax): Effects on stress and immune responses. Fish Shellfish Immunol. 2020, 100, 219-229. [CrossRef] [PubMed]

85. Zhai, B.; Zhang, C.; Yao, S.; Zhao, C.; He, X.; Xu, W.; Huang, K.; Luo, Y. Hypoglycemic and hypolipidemic effect of S-allyl-cysteine sulfoxide (alliin) in DIO mice. Sci. Rep. 2018, 8, 3527. [CrossRef] [PubMed]

86. Liu, C.T.; Hse, H.; Lii, C.K.; Chen, P.S.; Sheen, L.Y. Effects of garlic oil and diallyl trisulfide on glycemic control in diabetic rats. Eur. J. Pharmacol. 2005, 516, 165-173. [CrossRef]

87. Navarro, I.; Rojas, P.; Capilla, E.; Albalat, A.; Castillo, J.; Montserrat, N.; Codina, M.; Gutiérrez, J. Insights into insulin and glucagon responses in fish. Fish Physiol. Biochem. 2002, 27, 205-216. [CrossRef]

88. Moon, T.W. Glucose intolerance in teleost fish: Fact or fiction? Comp. Biochem. Physiol. B Biochem. Mol. Biol. 2001, 129, 243-249. [CrossRef]

89. Santos, G.; Schrama, J.; Mamauag, R.; Rombout, J.; Verreth, J. Chronic stress impairs performance, energy metabolism and welfare indicators in European seabass (Dicentrarchus labrax): The combined effects of fish crowding and water quality deterioration. Aquaculture 2010, 299, 73-80. [CrossRef]

90. Fought, E.; Vijayan, M.M. Mechanisms of cortisol action in fish hepatocytes. Comp. Biochem. Physiol. B Biochem. Mol. Biol. 2016, 199, 136-145. [CrossRef]

91. Barton, B.C. Stress in fishes: A diversity of responses with particular reference to changes in circulating corticosteroids. Integr. Comp. Biol. 2002, 42, 517-525. [CrossRef] [PubMed]

92. Kavitha, C.; Malarvizhi, A.; Senthil Kumaran, S.; Ramesh, M. Toxicological effects of arsenate exposure on hematological, biochemical and liver transaminases activity in an Indian major carp. Catla catla. Food Chem. Toxicol. 2010, 48, $2848-2854$. [CrossRef]

93. Yousefi, M.; Paktinat, M.; Mahmoudi, N.; Pérez-Jiménez, A.; Hoseini, S.M. Serum biochemical and non-specific immune responses of rainbow trout (Oncorhynchus mykiss) to dietary nucleotide and chronic stress. Fish Physiol. Biochem. 2016, 42, 1417-1425. [CrossRef] [PubMed]

94. Makvandi, H.; Khodadadi, M.; Keyvanshokoh, S.; Makvandi, Z.M. Effect of salinity stress on cortisol hormone and glucose in Grass carp fingerlings (Ctenopharyngodon idella). Int. J. Aquac. Fish Sci. 2012, 2, 77-84.

95. Odhiambo, E.; Angienda, P.O.; Okoth, P.; Onyango, D. Stocking density induced stress on plasma cortisol and whole blood glucose concentration in Nile tilapia fish (Oreochromis niloticus) of lake Victoria, Kenya. Int. J. Zool. 2020, 2020, 9395268. [CrossRef]

96. Dawood, M.A.; Eweedah, N.M.; El-Sharawy, M.E.; Awad, S.S.; Van Doan, H.; Paray, B.A. Dietary white button mushroom improved the growth, immunity, antioxidative status and resistance against heat stress in Nile tilapia (Oreochromis niloticus). Aquaculture 2020, 530, 735229. [CrossRef]

97. Adineh, H.; Naderi, M.; Afieh Nazer, A.; Yousefi, M.; Ahmadifar, E. Interactive effects of stocking density and dietary supplementation with Nano selenium and garlic extract on growth, feed utilization, digestive enzymes, stress responses, and antioxidant capacity of grass carp, Ctenopharyngodon Idella. J. World Aquac. Soc. 2020, 48, 372-378. [CrossRef]

98. Han, J.; Lawson, L.; Han, G.; Han, P. Spectrophotometric method for quantitative determination of allicin and total garlic thiosulfinates. Anal. Biochem. 1995, 225, 157-160. [CrossRef] [PubMed] 